International Journal of Modern Physics B Vol. 30, No. 32 (2016) 1699001 (28 pages) (c) World Scientific Publishing Company DOI: $10.1142 / \mathrm{S} 0217979216990010$

\title{
Author index Volume 30
}

Abadian, F., see MohsenNia, $M$.

Abadian, N., see Mohsen$\mathrm{Nia}, \mathrm{M}$.

Abadyan, M., see Abdi, J.

Abadyan, M., see MohsenNia, M.

Abbar, B., see Hamri, B.

Abbas, G., see Hussain, M. J.

Abbas, S. F., see Khashan, K. S.

Abdalla, S., Al-Marzouki, F. M. \& Abdel-Daiem, A. M., Nanopore formation by controlled electrical breakdown: Efficient molecular-sensors

Abdalla, S., see Batle, J.

Abdel-Daiem, A. M., see Abdalla, $\mathrm{S}$.

Abdel-Rahman, M. A., see Salah, M.

Abdel-Rahman, M., see Ilahi, B.

Abdel-Rahman, M., see Salah, M.

Abdellaoui, A., see Sahli, B. Abdi, J., Keivani, M. \& Abadyan, M., Microstructure-dependent dynamic stability analysis of torsional NEMS scanner in van der Waals regime

Abdullah, M., Proposal for a material with negative thermal expansion

Abdulrahman, S. A., see Ismail, R. A.

Abdulsattar, M. A., Elec-
B30 (2016) 1650148

B30 (2016) 1650148

B30 (2016) 1650109

B30 (2016) 1650148

B30 (2016) 1650076

B30 (2016) 1650161

B30 (2016) 1650080

B30 (2016) 1650185

B30 (2016) 1650123

B30 (2016) 1650185

B30 (2016) 1650110

B30 (2016) 1650210

B30 (2016) 1650110

B30 (2016) 1650230

B30 (2016) 1650109

B30 (2016) 1650238

B30 (2016) 1650094 tronic and spectroscopic properties of $\mathrm{Ge}$ nanocrystals using diamondoid structures: A density functional theory study

Abidri, B., see Sahli, B.

Abou-Aly, A. I., see ME. Barakat, M.

Abu Hilaleh, R., see Alrub, A. M.

Abutalib, M., see Batle, J.

Acosta Diaz, R. \& Svaiter, N. F., Finite-size effects in disordered $\lambda \varphi^{4}$ model

Acosta-Humánez, F., see Buchheit, R.

Adebambo, P. O., see Bamgbose, M. K.

Adebayo, G. A., see Bamgbose, M. K.

Adem, A. R., $A(2+1)$ dimensional Korteweg-de Vries type equation in water waves: Lie symmetry analysis; multiple expfunction method; conservation laws

Adetunji, B. I., Olayinka, A. S., Fashae, J. B. \& Ozebo, $\mathrm{V}$. C., Ab initio investigation of the electronic, lattice dynamic and thermodynamic properties of $\mathrm{ScCd}$ intermetallic alloy

Adhikari, N. P., see Bhandari, D.

Adhikari, N. P., see Pokharel, S.

Afshar, D., Motamedinasab, A., Anbaraki, A. \&
B30 (2016) 1650058

B30 (2016) 1650230

B30 (2016) 1650115

B30 (2016) 1650082

B30 (2016) 1650123

B30 (2016) 1650207

B30 (2016) 1650066

B30 (2016) 1650191

B30 (2016) 1650191

B30 (2016) 164000

B30 (2016) 1650175

B30 (2016) 1650064

B30 (2016) 1650205 
Jafarpour, M., Even and odd coherent states of supersymmetric harmonic oscillators and their nonclassical properties

Ahl Laamara, R., see El Qars, J.

Ahmad, I., see Ahmad, S.

Ahmad, I., see Hussain, M. J.

Ahmad, M., see Hussain, M. J.

Ahmad, R., see Ahmad, S.

Ahmad, S., Vaizie, H., Aliabad, H. A. R., Ahmad, R., Khan, I., Ali, Z., JalaliAsadabadi, S., Ahmad, I. \& Khan, A. A., Firstprinciples studies of pure and fluorine substituted alanines

Ahmadi, Z., Goudarzi, H. \& Jafari, A., Conduction band population in graphene in ultrashort strong laser field: Case of massive Dirac particles

Ahmed, R., see El Amine Monir, M.

Ahmed, W. K., see El Amine Monir, $\mathrm{M}$.

Akgol, O., see Bakir, M.

Akimov, M. L., Polyakov, P. A., Banishev, A. A. \& Usmanov, N. N., Detection of shape and sizes of magnetic impurity based on the shape of magnetic domain configuration

Akinlami, J. O., see Bamgbose, M. K.

Akriche, A., see Sahli, B.

Akutsu, H., see Imajo, S.

Akutsu, H., see Yamashita, $\mathrm{S}$.

Al Hazaa, A. N., see Ramay, S. M.

Al Shabaan, G. N., see Alrub, A. M.

Al, E. B., Ungan, F., Yesilgul, U., Kasapoglu, E., Sari, H. \& Sökmen, I., Infrared transitions between hydrogenic states in GaInNAs/GaAs quantum wells
B30 (2016) 1650026

B30 (2016) 1650134

B30 (2016) 1650079

B30 (2016) 1650161

B30 (2016) 1650161

B30 (2016) 1650079

B30 (2016) 1650079

B30 (2016) 1650122

B30 (2016) 1650034

B30 (2016) 1650034

B30 (2016) 1650133

B30 (2016) 1650081

B30 (2016) 1650191

B30 (2016) 1650230

B30 (2016) 1642014

B30 (2016) 1642007

B30 (2016) 1650020

B30 (2016) 1650082

B30 (2016) 1650139
Al-Douri, Y., see Benosman, F.

B30 (2016) 1650225

Al-Marzouki, F. M., see Abdalla, S.

Al-Omari, S., see Hamioud, $\mathrm{F}$.

B30 (2016) 1650185

B30 (2016) 1650031

Alahyarizadeh, Gh., see Goharrizi, A. Z.

Albertini, G., see Cardone, $\mathrm{F}$.

B30 (2016) 1650150

B30 (2016) 1550239

B30 (2016) 1650210

Alduraibi, M., see Ilahi, B.

AlGhamdi, G. S., see Hamioud, F.

B30 (2016) 1650031

Ali, A., see Hussain, M. J.

Ali, L. M., Simulation of $Q$ factor, bandgap frequency and defect band structure dependence upon hole radius of air formed in $I n_{x} G a_{1-x}$ As waveguides

Ali, M. A., Islam, A. K. M. A., Jahan, N. \& Karimunnesa, S., Firstprinciples study of $\mathrm{SnO}$ under high pressure

Ali, M. A., Roknuzzaman, M., Nasir, M. T., Islam, A. K. M. A. \& Naqib, S. H., Structural, elastic, electronic and optical properties of $\mathrm{Cu}_{3} \mathrm{MTe}_{4}(\mathrm{M}=\mathrm{Nb}$, Ta) sulvanites: An ab initio study

Ali, Z. I. \& Sango, M., A note on weak and strong probabilistic solutions for a stochastic quasilinear parabolic equation of generalized polytropic filtration

Ali, Z., see Ahmad, S.

Aliabad, H. A. R., see Ahmad, $S$.

Alisultanov, Z. Z., Berry phase and Landau levels in epitaxial graphene

Almanza, O., see Buchheit, R.

B30 (2016) 1640002

B30 (2016) 1650079

B30 (2016) 1650079

B30 (2016) 1650156

B30 (2016) 1650066

B30 (2016) 1650089

Alrub, A. M., Ong, L.H., Chew, K.-H., Khoshman, J. M., Al Shabaan, G. N. \& Abu Hilaleh, R., Phase transition of $\mathrm{BiMnO}_{3}$ multiferroic thin film by Landau-Ginzburg 
theory

Altinodotndal, Ş., see Kaya, A.

Aly, N. S., see ME. Barakat, M.

Amani, V., see Baniasadi, F.

Amellal, H., see Meslouhi, A.

Ananikian, N., see Lazaryan, $\mathrm{H}$.

Anashkina, E. I., Chichigina, O. A., Valenti, D., Kargovsky, A. V. \& Spagnolo, B., Predator population depending on lemming cycles

Anbaraki, A., see Afshar, D.

Anco, S. C. \& Khalique, C. M., Conservation laws of coupled semilinear wave equations

Anco, S. C., Symmetry properties of conservation laws

Anis, M., see Shejwal, N. N.

Anis-ur-Rehman, M., see Hussain, M. J.

Ansari, R., Rouhi, S. \& Aryayi, M., On the vibration of double-walled carbon nanotubes using molecular structural and cylindrical shell models

Arinodotkan, N., see Örnek, O.

Arjmandi, M. B., see Mahdian, $\mathbf{M}$.

Aryayi, M., see Ansari, R.

Asad, J. H., see Owaidat, M. Q.

Asgari, A., see Kashiri, M.

Asif Mahmood, see Mahmood, Q.

Asimakopoulos, A., Mogios, E. \& Xenikos, D. G., Geographic location, network patterns and population distribution of rural settlements in Greece

Atiq, S., see Ramay, S. M.

Awad, R., see ME. Barakat, M.

Aziz, A. \& Shoaib, M., Fluid structure interaction model for biological
B30 (2016) 1650082

B30 (2016) 1650090

B30 (2016) 1650115

B30 (2016) 1650174

B30 (2016) 1650061

B30 (2016) 1650135

B30 (2016) 1541003

B30 (2016) 1650026

B30 (2016) 1640004

B30 (2016) 1640003

B30 (2016) 1650159

B30 (2016) 1650161

B30 (2016) 1650007

B30 (2016) 1650169

B30 (2016) 1650107

B30 (2016) 1650007

B30 (2016) 1650166

B30 (2016) 1650121

B30 (2016) 1650160

B30 (2016) 1541009

B30 (2016) 1650020

B30 (2016) 1650115 systems in the presence of magnetic field

B30 (2016) 1640005

B30 (2016) 1640007

B30 (2016) 1650101

B30 (2016) 1650155

B30 (2016) 1650110

Badawi, E. A., see Salah, M.

Badmus, B. S., see Bamgbose, M. K.

Bagani, E., see Mohammad Alavi, S.

Bagdasaryan, A., see Batle, J.

Bahari, A., see Ranjbar, M.

Bahmad, L., see Jabar, A.

Bahmanpour, H., see Zahedi, E.

Bai, X., see Ying, C.

Bai, X.-M., Li, J.Q. \& Liang, J.-Q., Measurement-induced disturbance and thermal entanglement in a twoqutrit spin system with a uniaxial field

Bakir, M., Karaaslan, M., Dincer, F., Akgol, O. \& Sabah, C., Electromagnetic energy harvesting and density sensor application based on perfect metamaterial absorber

Balderas-Navarro, R. E., see González-Fernández, J. V.

Baltache, H., see El Amine Monir, M.

Băltăţeanu， D.-M. S., see Cotăescu, I. I.

Băltăţeanu, D.-M., see Cotăescu, I. I.

Bamgbose, M. K., Adebambo, P. O., Badmus, B. S., Dare, E. O., Akinlami, J. O. \& Adebayo, G. A., First-principles study of zinc-blende $B_{x} A l_{y} \operatorname{In}_{1-x-y} N$ quaternary alloy: Alchemical mixing approximation approach

Baniasadi, F., Sahraei, N., Fathi, M. B., Tehranchi, M. M., Safari, N. \& Amani, V., X-ray characterization of tripyridinium
B30 (2016) 1650191

B30 (2016) 1650050

B30 (2016) 1650123

B30 (2016) 1650182

B30 (2016) 1650233

B30 (2016) 1650101 B30 (2016) 1650131

B30 (2016) 1650171

B30 (2016) 1650133

B30 (2016) 1550248

B30 (2016) 1650034

B30 (2016) 1650190

B30 (2016) 1550245

B30 (2016) 1650191 
bis[tetrabromidoferrate(iii)] bromide asymmetric unit in solution by Debye function analysis

Banishev, A. A., see Akimov, M. L.

Bao, K., see Song, A.

Bao, X., see Yuan, G.

Barragán, V. E., Fortes, M., Solís, M. A. \& Salas, P., Bose gas in disordered, finite-layered systems

Basit, A., Murtaza, G., Mahmood, A., Yar, A. \& Muhammad, S., Structural, optoelectronic, and thermoelectric properties of $A Z n_{13}(A=N a, K, C a$, $\mathrm{Sr}, \mathrm{Ba}$ ) compounds

Bassani, D., see Cardone, F.

Batle, J., Bagdasaryan, A., Farouk, A., Abutalib, M. \& Abdalla, S., Quantum correlations in two coupled superconducting charge qubits

Bau, N. Q., see Hoi, B. D.

Benlamari, S., Korba, S. A., Lakel, S., Meradji, H., Ghemid, S. \& El Haj Hassan, F., Ab initio study of structural, mechanical, thermal and electronic properties of perovskites $\mathrm{Sr}(\mathrm{Li}, \mathrm{Pd}) \mathrm{H}_{3}$

Bennemann, K. H., Spindependent currents in nanostructures

Benosman, F., Dridi, Z., Al-Douri, Y. \& Bouhafs, B., Investigated electronic structure and magnetic ordering of rare earth impurities ( $\mathrm{Eu}, \mathrm{Gd})$ in $\mathrm{ZnO}$

Benyoussef, A., see Jabar, A.

Berović, M. B., see Rutonjski, M. S.

Besahraoui, F., Bouslama, M., Bouzaiene, L., Saidi, F., Maaref, H. \& Gendry, M., Thermal transfer and interaction mechanisms of localized excitons in families of InAs quantum
B30 (2016) 1650174

B30 (2016) 1650081

B30 (2016) 1650029

B30 (2016) 1650127

B30 (2016) 1650099

B30 (2016) 1650221

B30 (2016) 1550239

B30 (2016) 1650123

B30 (2016) 1650004

B30 (2016) 1650003

B30 (2016) 1642012

B30 (2016) 1650225

B30 (2016) 1650233

B30 (2016) 1550251 dashes grown on $\operatorname{InP}(001)$ vicinal substrate emitting near $1.55 \mu \mathrm{m}$ wavelength Bhalla, P., see Das, N.

Bhandari, D. \& Adhikari, N. P., Molecular dynamics study of diffusion of krypton in water at different temperatures

Bhattacharya, S., Comparison of decoherence and Zeno dynamics from the context of weak measurement for a two-level atom traversing through squeezed vacuum

Bian, B.-R., see Kong, X.K.

Bianucci, M., Nonconventional fluctuation dissipation process in nonHamiltonian dynamical systems

Bin Omran, S., see Chettri, $\mathrm{S}$.

Bin Omran, S., see El Amine Monir, M.

Bin Omran, S., see Kushwaha, A. K.

Boochani, A., see Sarmazdeh, M. M.

Bordag, M. \& Pirozhenko, I. G., Surface plasmon on graphene at finite $T$

Bordbar, G. H. \& Mohammadi Sabet, M. T., Magnetized liquid ${ }^{3} \mathrm{He}$ at $f$ nite temperature: A variational calculation approach

Borges, A. N., see Machado, P. C. M.

Bouafia, H., see Sahli, B.

Bouarissa, N., see Ferahtia, S.

Bouaza, A., see Sahli, B.

Bouazza, A. G., see Talha, N.

Bouazza, B., see Talha, N.

Bouhafs, B., see Benosman, F.

Bouhemadou, A., see El Amine Monir, M.

Bouslama, M., see Besahraoui, F.
B30 (2016) 1650145

B30 (2016) 1630015

B30 (2016) 1650064

B30 (2016) 1650016

B30 (2016) 1650070

B30 (2016) 1541004

B30 (2016) 1650078

B30 (2016) 1650034

B30 (2016) 1650018

B30 (2016) 1650117

B30 (2016) 1650120

B30 (2016) 1650129

B30 (2016) 1650218

B30 (2016) 1650230

B30 (2016) 1650147

B30 (2016) 1650230

B30 (2016) 1650153

B30 (2016) 1650153

B30 (2016) 1650225

B30 (2016) 1650034

B30 (2016) 1650145 
Bouzaiene, L., see Besahraoui, F.

Brito, C., see Duarte, R. N.

Bruzón, M. S., Classical and nonclassical symmetries of a generalized BenneyLuke equation

Buchheit, R., AcostaHumánez, F. \& Almanza, O., Electron paramagnetic resonance in $\mathrm{Cu}$ doped $\mathrm{ZnO}$

Çağatay, B. \& Ocak, S. B., An analytical solution for the graphene electronic spectrum in the presence of external fields and confinement potential

Cai, C. Z., see Cheng, W. D.

Cai, X.-Q., see Wang, T.-Y.

Cambui, D., see Iliass, T.

Campos, L. M. B. C., On the gravitational field of a radiating, isothermal perfect gas cloud

Cao, D.-W., Zhang, Y., Wang, J. \& Hu, Z.-D., Quantum coherence in a coupled-cavity array

Cao, Q.-L., see Wang, P.-P.

Cao, Z., see Liu, H.

Cardone, F., Albertini, G., Bassani, D., Cherubini, G., Guerriero, E., Mignani, R., Monti, M., Petrucci, A., Ridolfi, F., Rosada, A., Rosetto, F., Sala, V., Santoro, E. \& Spera, G., Nuclear metamorphosis in mercury

Carrim, A. H., Aziz, T., Mahomed, F. M. \& Khalique, C. M., Unsteady magnetohydrodynamic flow of a fourth grade fluid caused by an impulsively moving plate in a Darcy porous medium: A grouptheoretical analysis

Carvalho, P. R. S., All-loop order critical exponents for massless scalar field theory with Lorentz violation in the BPHZ method

Castro-García, R., see
B30 (2016) 1650145

B30 (2016) 1550261

B30 (2016) 1640006

B30 (2016) 1650066

B30 (2016) 1650062

B30 (2016) 1650052

B30 (2016) 1650178

B30 (2016) 1650002

B30 (2016) 1650038

B30 (2016) 1650114

B30 (2016) 1550250

B30 (2016) 1650033

B30 (2016) 1550239

B30 (2016) 1640007

B30 (2016) 1550259
González-Fernández, J. $\mathrm{V}$.

Çataltepe, O. A., New method to forecast the optimum lateral size for mesa structure in superconductors

Çetinkaya, H. G., see Kaya, A.

Chadli, R., see Kheffache, $\mathrm{S}$.

Chao, Z., see Wang, H.

Chatzigeorgiou, M., see Constantoudis, V.

Chen, B. S., see Zhang, S.

Chen, D.-Y., see Chen, S.-T.

Chen, H.-D., see Kong, S.$\mathrm{G}$.

Chen, L., see Chen, Y.

Chen, L., see Li, H.

Chen, L., see Li, Z.

Chen, L., see Tong, A.

Chen, M., see Shi, Y.

Chen, Q., see Xiao, W.-Z.

Chen, S., see Li, Z.

Chen, S., see Yuan, G.

Chen, S.-T., Li, Q., Chen, D.-Y. \& Cheng, J.-W., $N$-soliton-like and double Casoratian solutions of a nonisospectral AblowitzLadik equation

Chen, T. N., see Wang, X. P.

Chen, T., see Chen, Z.

Chen, T., see Song, A.

Chen, X. W., see de Ouyang, $\mathrm{S}$.

Chen, X. X., Zhao, M., Liu, G. Z., Yao, J. L. \& Gao, J., Effects of cerium substitution on magnetic properties of naturally layered $\mathrm{TbMn}_{6} \mathrm{Sn}_{6}$ compound

Chen, X., see Xiao, S.

Chen, X., see Yan, Y.

Chen, Y., Jiang, D., Gu, X., Xie, L. \& Chen, L., Interaction-free generation of orbital angular momentum entanglement

Chen, Y., see Li, P.

Chen, Y.-Y., see Guan, X.W.

Chen, Z., Yan, J., Kuang, Z., Chen, T. \& Li, D., Struc-
B30 (2016) 1550248

B30 (2016) 1650188

B30 (2016) 1650090

B30 (2016) 1650126

B30 (2016) 1650086

B30 (2016) 1541005

B30 (2016) 1650046

B30 (2016) 1640008

B30 (2016) 1550255

B30 (2016) 1650006

B30 (2016) 1650072

B30 (2016) 1650196

B30 (2016) 1650223

B30 (2016) 1650014

B30 (2016) 1650236

B30 (2016) 1650196

B30 (2016) 1650127

B30 (2016) 1640008

B30 (2016) 1650025

B30 (2016) 1650010

B30 (2016) 1650029

B30 (2016) 1650235

B30 (2016) 1650068 B30 (2016) 1650083

B30 (2016) 1650237

B30 (2016) 1650006 B30 (2016) 1650051

B30 (2016) 1630008 
tural, electronic and optical properties of hexagonal TaN compound

Cheng, J., see Li, P.

Cheng, J.-W., see Chen, S.$\mathrm{T}$.

Cheng, Q.-B., He, J., Yu, J., Zhao, X.-M. \& Kou, S.-P., Polygon sign rules of Majorana fermions in two-dimensional topological superconductors

Cheng, W. D., Cai, C. Z., Luo, Y., Li, Y. H. \& Zhao, C. J., Modeling and predicting the tensile strength of poly (lactic acid)/graphene nanocomposites by using support vector regression

Cheng, W., see Ding, D.

Cheng, Y., see Yang, K.

Cheng, Z., see Yang, X.-H.

Cherubini, G., see Cardone, F.

Chettri, S., Rai, D. P., Shankar, A., Khenata, R., Ghimire, M. P., Thapa, R. K. \& Bin Omran, S., $G G A+u$ and $m B J+u$ study of the optoelectronic, magnetic and thermoelectric properties of the $\mathrm{SmAlO}_{3}$ compound with spin-orbit coupling

Cheung, N., see Duarte, R. N.

Chew, K.-H., see Alrub, A. M.

Chichigina, O. A., see Anashkina, E. I.

Cohn, J. R., see Freericks, J. K.

Constantoudis, V., Kalimeri, M., Diakonos, F., Karamanos, K., Papadimitriou, C., Chatzigeorgiou, M. \& Papageorgiou, H., Longrange correlations and burstiness in written texts: Universal and languagespecific aspects

Cotăescu Jr, I.., see Cotăescu, I. I.

Cotăescu, I. I., Băltăţeanu,
B30 (2016) 1650010 B30 (2016) 1650051

B30 (2016) 1640008

B30 (2016) 1650213

B30 (2016) 1650052

B30 (2016) 1650028

B30 (2016) 1650168

B30 (2016) 1650222

B30 (2016) 1550239

B30 (2016) 1650078

B30 (2016) 1550261

B30 (2016) 1650082

B30 (2016) 1541003

B30 (2016) 1642001

B30 (2016) 1541005

B30 (2016) 1550245
D.-M. S. \& Cotăescu, Jr., I. I., Relativistic currents on ideal Aharonov-Bohm cylinders

Cotăescu, I. I., Băltăţeanu, D.-M. \& Cotăescu Jr, I.., Relativistic persistent currents in ideal AharonovBohm rings

Cotăescu, Jr., I. I., see Cotăescu, I. I.

Cuestas, E. \& Serra, P., Localization of the valence electron of endohedrally confined hydrogen, lithium and sodium in fullerene cages

Dadsetani, M., see Nejatipour, $\mathrm{H}$.

Dai, W., see Wang, T.

Dai, Y. Y., Wang, H., Yang, T. \& Zhang, Z. D., Resonant excitation of coupled skyrmions by spin-transfer torque

Dai, Y., Lin, Y., Zhao, H. \& Khalique, C. M., Global stability and Hopf bifurcation of a delayed computer virus propagation model with saturation incidence rate and temporary immunity

Dalhatu, S. A., Deraman, K. \& Hussin, R., Physical and optical properties of magnesium sulfoborate glasses doped $\mathrm{Dy}{ }^{3+}$ ions

Dang, T. M. H., see Tran, H. P.

Daoud, M., see El Qars, J.

Dare, E. O., see Bamgbose, M. K.

Das, A. K., see Mukherjee, P. K.

Das, N. \& Singh, N., Hotelectron relaxation in metals within the GötzeWölfle memory function formalism

Das, N., Bhalla, P. \& Singh, N., Memory function approach to correlated electron transport: A comprehensive review
B30 (2016) 1650190

B30 (2016) 1550245

B30 (2016) 1650190

B30 (2016) 1650055

B30 (2016) 1650077

B30 (2016) 1650092

B30 (2016) 1550254

B30 (2016) 1640009

B30 (2016) 1650054

B30 (2016) 1650195

B30 (2016) 1650134

B30 (2016) 1650191

B30 (2016) 1650053

B30 (2016) 1650071

B30 (2016) 1630015 
Das, S. \& Padhi, P. C., Effect of external magnetic field on the co-existence of superconductivity and antiferromagnetism in rare earth nickel borocarbides $\left(R \mathrm{Ni}_{2} \mathrm{~B}_{2} \mathrm{C}\right)$

de Melo, J. L., Pedrosa, I. A. \& Furtado, C., Coherent states of LandauAharonov-Casher levels de Ouyang, S., Mi, X. W., Zhou, Y. B., Chen, X. W. \& Song, K. H., Quantum phase transition and entanglement of one-dimensional spinless fermion model

de Sousa, J. R., see Neto, M. A.

de Souza, J. F. O. \& Furtado, C., On the confinement of massless Dirac fermions in topological Möbius strips

Deb, B., Finite-range model potentials for resonant interactions

Dehkordi, K. M., see Mohsen-Nia, M.

Deng, B., see Wang, J.

Deng, B., see Wei, X.

Deo, P. S., see Satpathi, U.

Deraman, K., see Dalhatu, S. A.

Dey, M. \& Maiti, S. K., Selective spin transport through a quantum heterostructure: Transfer matrix method

Diakonos, F., see Constantoudis, V.

Dincer, F., see Bakir, M.

Ding, D., Zheng, L., Wen, W. Z., Cheng, W. \& Qing, Z. Y., Effect of minor fe substitution for ni on the magnetic properties of a $\mathrm{Gd}_{55} \mathrm{Al}_{20} \mathrm{Ni}_{25}$ bulk metallic glass

Djumanov, S. H., see Yavidov, B.

Dong, H.-N., Liu, X.-S. \& Zhou, H.-F., The studies of the spin Hamilto- nian parameters and local structures for various $3 d^{3}$ hexacyanometallates of paramagnetic salts with diluents

B30 (2016) 1650044

Dong, J. M., see Feng, Y. L.

Dong, L., see Shi, X.

Dong, Y., see He, J.-Q.

Doorzad, L., see Yousefi, R.

Dougherty, R., see Ma, W.-

B30 (2016) 1650022 X.

Dress, A., Grünewald, S. \& Zeng, Z., A cognitive network for oracle bone characters related to animals

B30 (2016) 1650235

B30 (2016) 1630011

B30 (2016) 1650224

B30 (2016) 1650036

B30 (2016) 1650148

B30 (2016) 1550253

B30 (2016) 1550258

B30 (2016) 1550266

B30 (2016) 1650054

B30 (2016) 1650184

B30 (2016) 1541005

B30 (2016) 1650133

B30 (2016) 1650028

B30 (2016) 1650186
Dridi, Z., see Benosman, F.

Du, H., see Du, L. study of structural, electronic and thermodynamic properties of $\mathrm{Ba}_{2} \mathrm{YTaO}_{6}$

Du, Y., see Ying, C.

Duan, M. Y., see Xu, C.

Duarte, R. N., Faria, J. D., Brito, C., Veríssimo, N. C., Cheung, N. \& Garcia, A., Length scale of the dendritic microstructure affecting tensile properties of $\mathrm{Al}-(\mathrm{Ag})-(\mathrm{Cu})$ alloys

Duc, T. M., see Hoai, N. T. $\mathrm{X}$.

Egorova, V. P., see Grushevskaya, H. V.

Ekobena, H. P., see Sali, I.

El Allati, A., see Meslouhi, A.

El Amine Monir, M., Baltache, H., Khenata, R., Murtaza, G., Ahmed, R., Ahmed, W. K., Bin Omran, S. \& Bouhemadou, A., Half-metallicity and optoelectronic properties of $V$-doped zincblende $\mathrm{ZnS}$ and $\mathrm{CdS}$ alloys

El Baz, M., see Meslouhi, A.

El Baz, M., see Siyouri, F.

El Haj Hassan, F., see Benlamari, $S$.

El Qars, J., Daoud, M. \& Ahl Laamara, R., Nonclassi-
Du, L. \& Du, H., ab-initio
B30 (2016) 1650204

B30 (2016) 1650067

B30 (2016) 1650162

B30 (2016) 1550265

B30 (2016) 1650005

B30 (2016) 1640018

B30 (2016) 1630001

B30 (2016) 1650225

B30 (2016) 1650164

B30 (2016) 1650164

B30 (2016) 1650131

B30 (2016) 1650098

B30 (2016) 1550261

B30 (2016) 1650032

B30 (2016) 1642018

B30 (2016) 1550244

B30 (2016) 1650061

B30 (2016) 1650034

B30 (2016) 1650061

B30 (2016) 1650187

B30 (2016) 1650003 
cal correlations in a twomode optomechanical system

Fan, D. P., see Yang, P.

Fan, D., see Shi, Y.

Fan, H.-Y., see He, R.

Fan, J. D. \& Malozovsky, Y. M., Electron correlation effects beyond the random phase approximation

Fan, K., see Wu, S.

Fang, C., Quantitative calculation of barium vacancy formation mechanism effects on the roomtemperature resistance and PTCR effect in donordoped $\mathrm{BaTiO}_{3}$ ceramics

Fang, Y., Ni, Z. \& Yang, L., Optical waveguide switch through magnetic reflectance wall

Fareo, A. G. \& Mason, D. P., Group invariant solution for a pre-existing fracture driven by a power-law fluid in permeable rock

Faria, J. D., see Duarte, R. N.

Faridi, A., see Mahmood, Q. Farouk, A., see Batle, J.

Fashae, J. B., see Adetunji, B. I.

Fathi, M. B., see Baniasadi, F.

Fatima, A., Mahomed, F. M. \& Khalique, C. M., Noether symmetries and exact solutions of an Euler-Bernoulli beam model

Feng, S., Gao, D. \& Zhao, H., Nature of charge order in cuprate superconductors

Feng, Y. L., Zhu, J., Zhang, M., Gao, L. L., Liu, Y. F. \& Dong, J. M., The mean first passage time and stochastic resonance in gene transcriptional system with time delay

Ferahtia, S., Saib, S. \& Bouarissa, N., Thermodynamic properties of
B30 (2016) 1650134

B30 (2016) 1650045

B30 (2016) 1650014

B30 (2016) 1650009

B30 (2016) 1642006 B30 (2016) 1650208

B30 (2016) 1650211

B30 (2016) 1650119

B30 (2016) 1640010

B30 (2016) 1550261

B30 (2016) 1650160

B30 (2016) 1650123

B30 (2016) 1650175

B30 (2016) 1650174

B30 (2016) 1640011

B30 (2016) 1642005

B30 (2016) 1650067
ZnTe in zinc-blende and wurtzite phases

Filikhin, I., Karoui, A. \& Vlahovic, B., Single electron tunneling in double and triple quantum wells

Fleurov, V., see Vinish, Y.

Fortes, M., see Barragán, V. E.

Freericks, J. K., Cohn, J. R., van Dongen, P. G. J. \& Krishnamurthy, H. R., Infinite single-particle bandwidth of a Mott-Hubbard Insulator

Freitas, R. J. \& Shimakawa, $\mathrm{K}$., Kinetics of the persistent photocurrent in a-Si:H

Fridman, I., see Granstrom, C. R.

Fu, L., see Song, X.

$\mathrm{Fu}, \mathrm{T}$., see Li, Q.

$\mathrm{Fu}, \mathrm{X}$., see Hao, Y.

Fu, X., see Kong, Y.-H.

Furtado, C., see de Melo, J. L.

Furtado, C., see de Souza, J. F. O.

Gandarias, M. L. \& Rosa, M., Symmetries and conservation laws of a damped Boussinesq equation

Ganiyev, O., see Yavidov, B.

Gao, D., see Feng, S.

Gao, J., see Chen, X. X.

Gao, L. L., see Feng, Y. L.

Gao, N., Wu, J. H., Yu, L. \& Hou, H., Ultralow frequency acoustic bandgap and vibration energy recovery in tetragonal folding beam phononic crystal

Gao, P., see Liu, G.

Gao, T., see Yang, R.

Gao, X., Ren, H., Li, C., Wang, H. \& Tan, H., Firstprinciples study of the effect of lanthanum on the niobium diffusion in $\mathrm{fcc}$ $\mathrm{Fe}$

Garcia, A., see Duarte, R. N. Garibli, A., Huseynov, E.,
B30 (2016) 1650147

B30 (2016) 1642011

B30 (2016) 1650197

B30 (2016) 1650099

B30 (2016) 1642001

B30 (2016) 1650075

B30 (2016) 1642002

B30 (2016) 1650035

B30 (2016) 1650212

B30 (2016) 1650216

B30 (2016) 1650132

B30 (2016) 1650022

B30 (2016) 1650224

B30 (2016) 1640012

B30 (2016) 1650186

B30 (2016) 1642005

B30 (2016) 1650068

B30 (2016) 1650067

B30 (2016) 1650111

B30 (2016) 1650143

B30 (2016) 1550243

B30 (2016) 1650157

B30 (2016) 1550261 
Garibov, A. \& Mehdiyeva, R., Additive analysis of nano silicon under the influence of neutron irradiation

Garibov, A., see Garibli, A.

Gavrichkov, V. A., Pchelkina, Z. V., Nekrasov, I. A. \& Ovchinnikov, S. G., Pressure effect on the energy structure and superexchange interaction in undoped orthorhombic $\mathrm{La}_{2} \mathrm{CuO}_{4}$

Ge, M.-L., see Yu, L.-W.

Gendry, M., see Besahraoui, F.

Gervino, G., Mana, G. \& Palmisano, C., The ellipsoidal nested sampling and the expression of the model uncertainty in measurements

Getahun, S., Entanglement formulation in the framework of electrically pumped laser cavity

Ghalandari, M., Moving media: Second harmonic generation investigation

Ghemid, S., see Benlamari, $\mathrm{S}$.

Ghimire, M. P., see Chettri, $\mathrm{S}$.

Ghosh, S., see Satpathi, U.

Goharrizi, A. Z. \& Alahyarizadeh, Gh., The study of temperature effect on the performance characteristics of the InGaNbased vertical cavity surface emitting laser (VC$S E L)$ by solving the rate equations

Gong, Q., see Li, Z.

Gong, W.-J., see Yang, C.-J. González-Fernández, J. V., Herrera-Jasso, R., Ulloa-Castillo, N. A., Ortega-Gallegos, J., Castro-García, R., Lastras-Martínez, L. F., Lastras-Martínez, A., Balderas-Navarro, R. E., Mozume, T. \& Gozu, S.,
B30 (2016) 1650040 B30 (2016) 1650040

B30 (2016) 1650180

B30 (2016) 1630013

B30 (2016) 1650145

B30 (2016) 1541002

B30 (2016) 1650024

B30 (2016) 1650138

B30 (2016) 1650003

B30 (2016) 1650078

B30 (2016) 1550266

B30 (2016) 1650150

B30 (2016) 1650196

B30 (2016) 1650027
Residual electric fields of InGaAs/AlAs/AlAsSb (001) coupled double quantum wells structures assessed by photoreflectance anisotropy

Gorczyca-Goraj, A., see Janus-Zygmunt, I.

Goswami, P., Fermions on the low-buckled honeycomb structured lattice plane and classical Casimir-Polder force

Goudarzi, H., see Ahmadi, $\mathrm{Z}$.

Govinder, K. S., see M'pika Massoukou, R. Y.

Gozu, S., see GonzálezFernández, J. V.

Granstrom, C. R., Fridman, I., Lei, H.-C., Petrovic, C. \& Wei, J. Y. T., Pointcontact Andreev reflection spectroscopy on $\mathrm{Bi}_{2} \mathrm{Se}_{3}$ single crystals

Grusha, I., Menteshashvili, M. \& Japaridze, G. I., Effective Hamiltonian for a half-filled asymmetric ionic Hubbard chain with alternating on-site interaction

Grushevskaya, H. V. \& Krylov, G. G., Low frequency conductivity in monolayer graphene model with partial unfolding of Dirac bands

Grushevskaya, H. V., Krylova, N. G., Lipnevich, I. V., Orekhovskaja, T. I., Egorova, V. P. \& Shulitski, B. G., Enhancement of Raman light scattering in dye-labeled cell membrane on metalcontaining conducting polymer film

Grünewald, S., see Dress, A. $\mathrm{Gu}, \mathrm{H}$., see $\mathrm{Gu}, \mathrm{J}$.

Gu, J., Gu, H., Zhai, Y. \& Ma, P., Site occupation, phase stability, crystal and electronic struc-
B30 (2016) 1550248

B30 (2016) 1642013

B30 (2016) 1650087

B30 (2016) 1650122

B30 (2016) 1640022

B30 (2016) 1550248

B30 (2016) 1642002

B30 (2016) 1550260

B30 (2016) 1642009

B30 (2016) 1642018

B30 (2016) 1630001

B30 (2016) 1650165 
tures of the doped $S$ phase $\left(\mathrm{Al}_{2} \mathrm{CuMg}\right)$

Gu, X. Q., see Li, S. P.

$\mathrm{Gu}, \mathrm{X}$., see Chen, Y.

Gu, Y., see Shi, Y.

Guan, X.-W. \& Chen, Y.-Y., Yang-Yang equilibrium statistical mechanics: A brilliant method

Guerriero, E., see Cardone, $\mathrm{F}$.

Guo, B.-X. \& Lin, J., Exact soliton solutions for the interaction of fewcycle-pulse with nonlinear medium

Guo, L., Jiang, L. \& Zhou, Y., Impact of interface misfit strain on the movement and tilt angles of the domain wall in ferroelectric thin films

Guo, Y., see Wei, J.

Gupta, V. \& Singh, A. K., Scaling laws of gelatin hydrogels for steady $d y$ namic friction

Gül, Y., Synchronization of networked Jahn-Teller systems in SQUIDs

Gómez-Arias, W. A. \& Naumis, G. G., Analytical calculation of electron group velocity surfaces in uniform strained graphene

Li, H.-M., see Kong, X.-K.

Hallouche, A., see Hamri, B.

Hamerly, R., Inaba, K., Inagaki, T., Takesue, H., Yamamoto, Y. \& Mabuchi, $\mathrm{H}$., Topological defect formation in $1 D$ and $2 D$ spin chains realized by network of optical parametric oscillators

Hamioud, F., AlGhamdi, G. S., Al-Omari, S. \& Mubarak, A. A., Ab initio investigation of the structural, electronic, magnetic and optical properties of the perovskite $\mathrm{TlMnX}_{3}$ (X $=\mathrm{F}, \mathrm{Cl}$ ) compounds
B30 (2016) 1650165

B30 (2016) 1650140

B30 (2016) 1650006

B30 (2016) 1650014

B30 (2016) 1630008

B30 (2016) 1550239

B30 (2016) 1640013

B30 (2016) 1650173

B30 (2016) 1650049

B30 (2016) 1650198

B30 (2016) 1650125

B30 (2016) 1550263

B30 (2016) 1650070

B30 (2016) 1650076

B30 (2016) 1630014

B30 (2016) 1650031
Hamri, A., see Hamri, B.

B30 (2016) 1650076

Hamri, A., see Hamri, B.

Hamri, B., Hamri, A., Hamri, A., Hallouche, A. \& Abbar, B., First principle study of a new halfmetallic ferrimagnetic full-Heusler alloy $\mathrm{Ti}_{2} \mathrm{VPb}$

Han, C., see Wei, X.

Han, R., see Wang, J.

Han, X.-Y., Peng, Y.-F. \& Ma, Z.-J., The electric conductivity characteristics of seawater ionic solution under the influence of magnetic field

Han, Z., see Luo, S.

Hao, Y., Song, Y. \& Fu, X., The properties of TonksGirardeau gas at finite temperature and comparison with spin-polarized fermions

Hassan, M., see Mahmood, Q.

Hassouni, Y., see Meslouhi, A.

Hassouni, Y., see Siyouri, F.

He, D.-X., see Liu, X.

He, J., see Cheng, Q.-B.

He, J., see Liu, G.

He, J., see Wei, J.

He, J.-Q., Dong, Y., Zhang, F.-D., Yu, Y.-J., Jin, G.-Y. \& Liu, L.-D., The spacedependent model and output characteristics of intra-cavity pumped dualwavelength lasers

He, M.-D., see Wang, K.-J.

He, R. \& Fan, H.-Y., Evolution of l-photon excited thermo vacuum state in a single-mode damping channel

He, Y., see Yang, K.

Hernández-Ortiz, S., Valenzuela, D., Raya, A. \& Sánchez-Madrigal, S., Light absorption in distorted graphene

Herrera-Jasso, R., see González-Fernández, J. V.

Hiadsi, S., see Sahli, B.
B30 (2016) 1650076

B30 (2016) 1650076

B30 (2016) 1550258

B30 (2016) 1550253

B30 (2016) 1650172

B30 (2016) 1650017

B30 (2016) 1650216

B30 (2016) 1650160

B30 (2016) 1650061

B30 (2016) 1650187

B30 (2016) 1650130

B30 (2016) 1650213

B30 (2016) 1650143

B30 (2016) 1650049

B30 (2016) 1550265

B30 (2016) 1650088

B30 (2016) 1650009

B30 (2016) 1650168

B30 (2016) 1650084

B30 (2016) 1550248

B30 (2016) 1650230 
Hoai, N. T. X. \& Duc, T. M., Nonclassical properties and teleportation in the two-mode photonadded displaced squeezed states

Hoc, N. Q., see Tinh, B. D.

Hoi, B. D., Bau, N. Q. \& Nam, N. D., Investigation of the magnetoresistivity in compositional superlattices under the influence of an intense electromagnetic wave

Hong, N. V., see Nhan, N. T. $\mathrm{T}$.

Hong, S.-K., see Yoon, J. S.

Horchani, R., Cold molecules: Formation, rovibrational cooling and electronic conversion

Hou, F., see Li, D.

Hou, H., see Gao, N.

Hou, Q., Li, W., Xu, Z. \& Zhao, C., Study on the effect of high $V$ doping on the conductivity of anatase $\mathrm{TiO}_{2}$

Hou, Q., Li, Y., Qu, L. \& Zhao, C., The effect of $C d$ substitution doping on the bandgap and absorption spectrum of $\mathrm{ZnO}$

Hou, Q., see Ying, C.

Hristopulos, D. T. \& Tsantili, I. C., Space-time models based on random fields with local interactions

$\mathrm{Hu}$, B., see Li, D.

Hu, J., Ke, Q. \& Ji, Y., Lyapunov-based state transfer and maintenance for non-Markovian quantum system

Hu, Z., see Zhu, Y.

Hu, Z.-D., see Cao, D.-W.

Huang, C., see Zhong, M.M.

Huang, J., see Meng, X.

Huang, J., see Shi, Y.

Huang, L., Wang, G., Wang, Y., Pang, W. \& Ma, Q., A link density clustering algorithm based on automatically selecting den-

sity peaks for overlapping community detection

Huang, L., see Wang, G.

Huang, S., see Song, H.

Huang, Y., Lu, X., Liang,

B30 (2016) 1650032

B30 (2016) 1550267

B30 (2016) 1650004

B30 (2016) 1650059

B30 (2016) 1650146

B30 (2016) 1630010

B30 (2016) 1650012

B30 (2016) 1650111

B30 (2016) 1650001

B30 (2016) 1650215

B30 (2016) 1650131

B30 (2016) 1541007 B30 (2016) 1650012

B30 (2016) 1650177

B30 (2016) 1650193

B30 (2016) 1650114

B30 (2016) 1650201

B30 (2016) 1650019

B30 (2016) 1650014
G. \& Xu, Z., Pentamodal behaviors and acoustic bandgaps of asymmetric pentamode elastic metamaterials

Huang, Y., see Liu, Y.

Huang, Y.-J., see Yang, X.$\mathrm{H}$.

Hung, P. K., see Nhan, N. T. $\mathrm{T}$.

Huseynov, E., see Garibli, A.

Hussain, M. J., Raza, R., Ahmad, M., Ali, A., Ahmad, I., Syed, W. A. A., Janjua, N. K., Anis-ur-Rehman, M., Khan, M. A., Shahid, S. A. \& Abbas, G., Electrochemical study of natural gas fueled electrodes for low temperature solid oxide fuel cell

Hussaini, S. S., see Shejwal, N. N.

Hussin, R., see Dalhatu, S. A.

Hutchinson, A. J., see Kokela, L. N.

Ibrahim, S., see ME. Barakat, M.

Ilahi, B., Abdel-Rahman, M., Zaaboub, Z., Zia, M. F., Alduraibi, M. \& Maaref, H., Thermal annealing induced multiple phase in $\mathrm{V} / \mathrm{V}_{2} \mathrm{O}_{5}$ alternating multilayer structure

Iliass, T. \& Cambui, D., The combined effect of attraction and orientation zones in $2 D$ flocking models

Imajo, S., Yamashita, S., Akutsu, H. \& Nakazawa, Y., Quadratic temperature dependence of electronic heat capacities in the $\kappa$-type organic superconductors

B30 (2016) 1642014

Inaba, K., see Hamerly, R.

Inagaki, T., see Hamerly, R.
B30 (2016) 1650167

B30 (2016) 1650023

B30 (2016) 1650113

B30 (2016) 1650118

B30 (2016) 1650085

B30 (2016) 1650222

B30 (2016) 1650059

B30 (2016) 1650040

B30 (2016) 1650161

B30 (2016) 1650159

B30 (2016) 1650054

B30 (2016) 1640014

B30 (2016) 1650115

B30 (2016) 1650210

B30 (2016) 1650002

B30 (2016) 1630014

B30 (2016) 1630014 
Ingle, N. B., see Taide, S. T. Iqbal, M. A., see Ullah, N.

Iqbal, Y., see Muhammad, $\mathrm{R}$.

Islam, A. K. M. A., see Ali, M. A.

Islam, A. K. M. A., see Ali, M. A.

Ismail, R. A., Sulaiman, G. M. \& Abdulrahman, S. A., Preparation of iron oxide nanoparticles by laser $a b$ lation in DMF under effect of external magnetic field

Jabar, A., Tahiri, N., Bahmad, L. \& Benyoussef, A., Magnetic properties of a tri-decorated graphene structure: Monte Carlo study

Jafari, A., see Ahmadi, Z.

Jafarpour, M., see Afshar, D.

Jahan, N., see Ali, M. A.

Jalali-Asadabadi, S., see Ahmad, S.

Janjua, N. K., see Hussain, M. J.

Janus-Zygmunt, I., Kȩdzierska, B., GorczycaGoraj, A., Zipper, E. \& Maśka, M. M., Quantum dot-ring nanostructure A comparison of different approaches

Japaridze, G. I., see Grusha, I.

Jaya, N. V., see Marselin, M. A.

Jayannavar, A. M., see Pal, P. $\mathrm{S}$.

Jayoti, D., see Singh, A.

Ji, Y., see Hu, J.

Jia, J., see Zhang, X.-K.

Jia, L., see Tian, Z.

Jia, Y. \& Liu, J., Exciton effects in strained armchair graphene nanoribbons

Jiang, D., see Chen, Y.

Jiang, J., see Yang, L.-X.

Jiang, L., see Guo, L.

Jiang, P., see Song, A.

Jiang, P., see Wang, X. P.

Jiang, P., see Wang, X. P.
B30 (2016) 1650179

B30 (2016) 1650137

B30 (2016) 1650104

B30 (2016) 1650089

B30 (2016) 1650228

B30 (2016) 1650094

B30 (2016) 1650233

B30 (2016) 1650122

B30 (2016) 1650026

B30 (2016) 1650228

B30 (2016) 1650079

B30 (2016) 1650161

B30 (2016) 1642013

B30 (2016) 1550260

B30 (2016) 1650056

B30 (2016) 1650219

B30 (2016) 1650011

B30 (2016) 1650177

B30 (2016) 1650093

B30 (2016) 1550264

B30 (2016) 1650021

B30 (2016) 1650006

B30 (2016) 1550252

B30 (2016) 1650173

B30 (2016) 1650029

B30 (2016) 1650203

B30 (2016) 1650025
Jiang, S., see Liu, H.

B30 (2016) 1650229

Jiang, Y.-Q., see Kong, Y.$\mathrm{H}$.

Jiao, P., see Wang, T.

Jin, G.-Y., see He, J.-Q.

Jin, L.-H., see Yang, C.-J.

Jin, W., see Wang, G.

Jin, Y. \& Niu, S., Stability of a Beddington-DeAngelis type predator-prey model with trichotomous noises

Jing, Y., see Liu, D.

Jing, Y., see Li, D.

John Peter, A., see Sujanah, $\mathrm{P}$.

Kadoun, A.-E.-D., see Talha, N.

Kagan, A. I., see Lushnikov, A. A.

Kalimeri, M., see Constantoudis, $\mathrm{V}$.

Kaneko, S., Murai, D., Sh. Fujii \& Kiguchi, M., Surface enhanced Raman scattering of single 1,4benzenedithiol molecular junction

Kaneyoshi, T., Ferrimagnetic behaviors in a transverse Ising nanoisland

Karaaslan, M., see Bakir, M.

Karamanos, K., see Constantoudis, V.

Kargovsky, A. V., see Anashkina, E. I.

Karimunnesa, S., see Ali, M. A.

Karoui, A., see Filikhin, I.

Kasapoglu, E., see Al, E. B.

Kashiri, M. \& Asgari, A., Evaluating the effects of nonlinear optical gain and thermal carrier escape on the performance of InGAas/GaAs selfassembled quantum dot lasers

Kato, S., see Mahudeswaran, A.

B30 (2016) 1650132

B30 (2016) 1650092

B30 (2016) 1550265

B30 (2016) 1650027

B30 (2016) 1650096

B30 (2016) 1650102

B30 (2016) 1650037

B30 (2016) 1650012

B30 (2016) 1650069

B30 (2016) 1650153

B30 (2016) 1541008

B30 (2016) 1541005

B30 (2016) 1642010

B30 (2016) 1650073

B30 (2016) 1650133

B30 (2016) 1541005

B30 (2016) 1541003

B30 (2016) 1650228

B30 (2016) 1642011

B30 (2016) 1650139

B30 (2016) 1650121

B30 (2016) 1650008

Kaya, A., Çetinkaya, H. G., Altinodotndal, Ş. \& Uslu, I., A comparative study on the electrical parameters of Au/n-Si Schottky diodes with and without inter- 
facial $\left(\mathrm{Ca}_{1.9} \operatorname{Pr}_{0.1} \mathrm{Co}_{4} \mathrm{O}_{x}\right)$ layer

Ke, Q., see Hu, J.

Kȩdzierska, B., see JanusZygmunt, I.

Keivani, M., see Abdi, J.

Keivani, M., see Mohsen$\mathrm{Nia}, \mathrm{M}$.

Khalique, C. M., see Anco, S. C.

Khalique, C. M., see Carrim, A. $\mathrm{H}$.

Khalique, C. M., see Dai, Y.

Khalique, C. M., see Fatima, A.

Khalique, C. M., see Molati, M.

Khalique, C. M., see Zhang, L.

Khan, A. A., see Ahmad, S.

Khan, I., see Ahmad, S.

Khan, M. A., see Hussain, M. J.

Khan, N. A. \& Muzaffar, M. U., Decoupling of the $\mathrm{CuO}_{2}$ plane and superconductivity in $\mathrm{Cu}_{0.5} \mathrm{Tl}_{0.5} \mathrm{Ba}_{2}\left(\mathrm{Ca}_{2-y} \mathrm{Sr}_{y}\right)_{\text {- }}$ $\mathrm{Cu}_{3} \mathrm{O}_{10-\delta}(y=0-0.4)$ samples

Khan, N. A., see Muzaffar, M. U.

Khan, S., see Singh, R. J.

Khashan, K. S. \& Abbas, S. F., Characterization of InN nanoparticles prepared by laser as photodetector

Khater, A., see Kheffache, S.

Kheffache, S., Chadli, R. \& Khater, A., Vibrational properties at the ordered metallic surface alloy system $\mathrm{Au}(110)-1 \times 2-\mathrm{Pd}$

Khenata, R., see Chettri, S.

Khenata, R., see El Amine Monir, $\mathrm{M}$.

Khenata, R., see Kushwaha, A. $\mathrm{K}$.

Khenata, R., see Ullah, N.

Khomami, M. M. D., Rezvanian, A. \& Meybodi, M. R., Distributed learning automata-based al-
B30 (2016) 1650090

B30 (2016) 1650177

B30 (2016) 1642013

B30 (2016) 1650109

B30 (2016) 1650148

B30 (2016) 1640004

B30 (2016) 1640007

B30 (2016) 1640009

B30 (2016) 1640011

B30 (2016) 1640021

B30 (2016) 1640029

B30 (2016) 1650079

B30 (2016) 1650079

B30 (2016) 1650161

B30 (2016) 1650097

B30 (2016) 1650112

B30 (2016) 1650170

B30 (2016) 1650080

B30 (2016) 1650126

B30 (2016) 1650126

B30 (2016) 1650078

B30 (2016) 1650034

B30 (2016) 1650018

B30 (2016) 1650137 gorithm for community detection in complex networks

Khoshman, J. M., see Alrub, A. M.

Kien, P. H., see Nhan, N. T. $\mathrm{T}$.

Kiguchi, M., see Kaneko, S.

Kislov, A. N., Vibrational dynamics of crystalline silicon dioxide with charged Ge impurities

Koblischka, M. R. \& Muralidhar, M., Pinning force scaling analysis of $\mathrm{Fe}$ based high- $T_{c}$ superconductors

Kofané, T. C., see Sali, I.

Kojima, T., see Mahudeswaran, A.

Kokela, L. N., Mason, D. P. \& Hutchinson, A. J., Conservation laws and conserved quantities for the two-dimensional laminar wake

Kolovsky, A. R., BoseHubbard Hamiltonian: Quantum chaos approach

Kong, S.-G., Chen, H.-D., Torda, A. \& Lee, H. C., Genomes: At the edge of chaos with maximum information capacity

Kong, X.-K., Mo, J.-J., Yu, Z.-Y., Shi, W., H.-M. Li \& Bian, B.-R., Reconfigurable designs for electromagnetically induced transparency in solid state plasma metamaterials with multiple transmission windows

Kong, Y., Liu, L., Xia, S., Wang, H. \& Wang, M., First-principle study on electronic structure and optical properties of GaN nanowires with different cross-sections

Kong, Y., see Xia, S.

Kong, Y.-H., Jiang, Y.-Q., Fu, X. \& Li, A.-H., Manipulable MR effect in a $\delta$-doped magnetic nanos-
B30 (2016) 1650042

B30 (2016) 1650082

B30 (2016) 1650059

B30 (2016) 1642010

B30 (2016) 1650206

B30 (2016) 1630017

B30 (2016) 1550244

B30 (2016) 1650008

B30 (2016) 1640014

B30 (2016) 1630009

B30 (2016) 1550255

B30 (2016) 1650070

B30 (2016) 1650136

B30 (2016) 1650217 
tructure

Konishi, E., Core-halo quasi-stationary states in the Hamiltonian meanfield model

Korba, S. A., see Benlamari, S.

Kornilovitch, P., Periodically driven adiabatic bipolarons

Kosterlitz, J. M. \& Thouless, D. J., Early work on defect driven phase transitions

Kou, S.-P., see Cheng, Q.-B.

Kozlovskii, M. P., see Pylyuk, I. V.

Kregar, A. \& Ramšak, A., Exact unitary transformation for Rashba Rings in magnetic and electric fields

Krishnamurthy, H. R., see Freericks, J. K.

Kristoffel, N. \& Rubin, P., Multiband superconductivity with competing intra- and interband pair channels

Kruchinin, S. P., see Vlaskina, S. I.

Kruchinin, S. P., Energy spectrum and wavefunction of electrons in hybrid superconducting nanowires

Kruchinin, S., Zolotovsky, A., Yamashita, S. \& Nakazawa, Y., Thermodynamics of the $d$-wave pairing in organic superconductors

Krylov, G. G., see Grushevskaya, H. V.

Krylova, N. G., see Grushevskaya, H. V.

Kuang, Z., see Chen, Z.

Kurbanov, U., see Yavidov, B.

Kushwaha, A. K., Khenata, R. \& Bin Omran, S., Vibrational, mechanical and thermodynamical properties of indium thiospinels $\mathrm{MIn}_{2} \mathrm{~S}_{4}(\mathrm{M}=\mathrm{Cd}, \mathrm{Zn}$ and $\mathrm{Mg}$ )
B30 (2016) 1650132

B30 (2016) 1630007

B30 (2016) 1650003

B30 (2016) 1650105

B30 (2016) 1630018

B30 (2016) 1650213

B30 (2016) 1650232

B30 (2016) 1642016

B30 (2016) 1642001

B30 (2016) 1642017

B30 (2016) 1642019

B30 (2016) 1642008

B30 (2016) 1642020

B30 (2016) 1642009

B30 (2016) 1642018

B30 (2016) 1650010

B30 (2016) 1650186

B30 (2016) 1650018
Lai, G.-Z., Liang, X. \& Lv, J., The optical dielectric model of $\mathrm{Cu}_{2} \mathrm{O}$ thin film and its verification

B30 (2016) 1550247

B30 (2016) 1650003

Lakel, S., see Benlamari, S.

Lastras-Martínez, A., see González-Fernández, J. $\mathrm{V}$.

Lastras-Martínez, L. F., see González-Fernández, J. $\mathrm{V}$.

Lazaryan, H., Nalbandyan, M. \& Ananikian, N., Diamond chain with delocalized interstitial spins: Magnetization, thermal and entanglement properties

Le, V. H., see Tran, H. P.

Lee, C. W., see Sujanah, P.

Lee, H. C., see Kong, S.-G.

Lee, T., Chiral fermion and boundary state formulation: Resonant pointcontact tunneling

Leggett, A. J., Majorana fermions in condensedmatter physics

Lei, H.-C., see Granstrom, C. R.

Lei, L., see Li, H.

Lei, X., see Zhong, S.

Lenarčič, Z., see Prelovšek, $P$.

Li, A.-H., see Kong, Y.-H.

Li, C., see Gao, X.

Li, D., Hu, B., Wang, J., Jing, Y. \& Hou, F., Coherence resonance in the twodimensional neural map driven by non-Gaussian colored noise

B30 (2016) 1650012

Li, D., see Chen, Z.

Li, D., see Li, S.

Li, D.-J., see Tang, B.

Li, F., see Li, Z. J.

Li, H., Wang, B., Pei, H., Shu, W., Chen, L., Lei, L. \& Zhou, J., Reflective three-port high-efficiency grating with two dielectric layers based on a sandwiched configuration

Li, J. \& Zhu, W., Dynamics of solutions of some
B30 (2016) 1650135

B30 (2016) 1650195

B30 (2016) 1650069

B30 (2016) 1550255

B30 (2016) 1650047

B30 (2016) 1630012

B30 (2016) 1642002

B30 (2016) 1650072

B30 (2016) 1650176

B30 (2016) 1642015

B30 (2016) 1650132

B30 (2016) 1650157

B30 (2016) 1650010

B30 (2016) 1550240

B30 (2016) 1650100

B30 (2016) 1550242

B30 (2016) 1650072 
travelling wave systems determined by integrable Liénard system

Li, J., Zheng, D.-Q. \& Zhong, W.-R., Thermal properties of defective fullerene

Li, J., see Li, S.

Li, J.-B., see Wang, K.-J.

Li, J.-Q., see Bai, X.-M.

Li, P., Cheng, J., Chen, Y. \& Wang, H., A model of social network formation under the impact of structural balance

Li, Q., Fu, T., Peng, T., Peng, X., Liu, C. \& Shi, X., Coalescence of $\mathrm{Cu}$ contacted nanoparticles with different heating rates: A molecular dynamics study

Li, Q., see Chen, S.-T.

$\mathrm{Li}, \mathrm{Q}$., see $\mathrm{Xu}, \mathrm{C}$.

Li, Q.-F., see Wang, L.

Li, S. P. \& Gu, X. Q., Temperature-dependent photoluminescence spectra of $\mathrm{ZnO}$ nanorod arrays grown on transparent conducting substrates by hydrothermal routes

Li, S., Li, D., Wang, J. \& Yu, $\mathrm{H}$., Iterative learning control algorithm for spiking behavior of neuron model

Li, S., Yan, H. \& Li, J., Synchronization of complex networks coupled by periodically intermittent noise

Li, W., see Hou, Q.

Li, Y. H., see Cheng, W. D.

Li, Y. X, see Yang, P.

Li, Y., see Hou, Q.

Li, Y., see Tong, A.

Li, Y., see Zhu, Y.

Li, Z. J., Li, F. \& Zhang, L. L., Controlling soliton collision behaviors in the condensates by the height of barrier potential

Li, Z., Wang, H., Chen, L., Chen, S. \& Gong, Q., The electron-longitudinal

optical phonon scattering rate in GaInAsP/InP

B30 (2016) 1640015

B30 (2016) 1650194

B30 (2016) 1650057

B30 (2016) 1650088

B30 (2016) 1650171

B30 (2016) 1650051

B30 (2016) 1650212

B30 (2016) 1640008

B30 (2016) 1650098

B30 (2016) 1650116

B30 (2016) 1650140

B30 (2016) 1550240

B30 (2016) 1650057

B30 (2016) 1650001

B30 (2016) 1650052

B30 (2016) 1650045

B30 (2016) 1650215

B30 (2016) 1650223

B30 (2016) 1650193

B30 (2016) 1550242 stepped quantum well

Li, Z.-J., see Wang, K.-J.

Li, H.-M., see Kong, X.-K.

Liang, G., see Huang, Y.

Liang, J.-Q., see Bai, X.-M.

Liang, S., see Shi, X.

Liang, X., see Lai, G.-Z.

Lim, C. \& Zhang, W., Social opinion dynamics is not chaotic

Lin, J., see Guo, B.-X.

Lin, L., see Ying, C.

Lin, Y., see Dai, Y.

Ling, F., see Yang, X.-H.

Lipnevich, I. V., see Grushevskaya, H. V.

Liu, C. M., see Xu, C.

Liu, C., see Li, Q.

Liu, D., Wang, C. \& Jing, Y., Estimating the optimal number of communities by cluster analysis

Liu, G. Z., see Chen, X. X.

Liu, G., He, J., Luo, K., Gao, P. \& Ma, L., Scale-free networks of the earth's surface

Liu, H., Yang, Q., Yang, M. \& Cao, Z., Improving entanglement of two atoms in strong coupling regime

Liu, H., Zhang, Z., Wu, Y., Jiang, S. \& Yu, C., Isolated attosecond pulses generation from coherent superposition state of helium ion in static electric fields and spatial nonhomogeneous fields

Liu, J., see Jia, Y.

Liu, J., see Shen, J.-M.

Liu, L., see Kong, Y.

Liu, L., see Xia, S.

Liu, L.-D., see He, J.-Q.

Liu, L.-H., see Wang, K.-J.

Liu, M., see Yan, Y.

Liu, Q., see Zhang, B.

Liu, R., see Shi, X.

Liu, X., Wei, Y.-M., Wang, J., Wang, W.-J., He, D.X. \& Song, Z.-J., Community detection enhancement using non-negative
B30 (2016) 1650196

B30 (2016) 1650088

B30 (2016) 1650070

B30 (2016) 1650118

B30 (2016) 1650171

B30 (2016) 1650162

B30 (2016) 1550247

B30 (2016) 1541006

B30 (2016) 1640013

B30 (2016) 1650131

B30 (2016) 1640009

B30 (2016) 1650222

B30 (2016) 1642018

B30 (2016) 1650098

B30 (2016) 1650212

B30 (2016) 1650037

B30 (2016) 1650068

B30 (2016) 1650143

B30 (2016) 1650033

B30 (2016) 1650229

B30 (2016) 1650021

B30 (2016) 1550256

B30 (2016) 1650136

B30 (2016) 1650217

B30 (2016) 1550265

B30 (2016) 1650088

B30 (2016) 1650237

B30 (2016) 1650013

B30 (2016) 1650162 
matrix factorization with graph regularization

Liu, X., see Song, H.

Liu, X.-J., see Yang, L.-X. Liu, X.-S., see Dong, H.-N. Liu, Y. F., see Feng, Y. L.

Liu, Y., Huang, Y., Xiao, Z., Yang, C. \& Reng, X., First principles calculations of formation energies and elastic constants of inclusions $\alpha-\mathrm{Al}_{2} \mathrm{O}_{3}, \mathrm{MgO}$ and AlN in aluminum alloy

Liu, Y., see Xiao, S.

Lone, M. Q. \& Yarlagadda, S., Decoherence dynamics of interacting qubits coupled to a bath of local optical phonons

Longhi, S., Accelerated and Airy-Bloch oscillations

Lou, K., Wang, F. H., Lu, Y. J. \& Zhao, X., Effect of inhomogeneous re-oxidation on $\mathrm{Ni}$-based SOFC oxidation resistance

Lu, M., see Wei, X.

Lu, M., see Wei, X.

Lu, X., see Huang, Y.

Lu, Y. J., see Lou, K.

Lu, Z.-L., see Zhang, X.-K.

Lui, Z., see Wang, H.

Luo, H., see Luo, S.

Luo, K., see Liu, G.

Luo, L. \& Zhao, P., The hamiltonian structure and quasi-periodic solutions for the generalized associated Camassa-Holm equation

Luo, S., Luo, H. \& Han, Z., Acoustic emission study of deformation behavior of nacre

Luo, Y., see Cheng, W. D.

Lushnikov, A. A. \& Kagan, A. I., A linear model of population dynamics

Lv, J., see Lai, G.-Z.

M'pika Massoukou, R. Y. \& Govinder, K. S., Symmetry analysis for hyperbolic equilibria using a TB/dengue fever model
B30 (2016) 1650130

B30 (2016) 1650113

B30 (2016) 1550252

B30 (2016) 1650204

B30 (2016) 1650067

B30 (2016) 1650085

B30 (2016) 1650083

B30 (2016) 1650063

B30 (2016) 1650189

B30 (2016) 1650200

B30 (2016) 1650142

B30 (2016) 1550258

B30 (2016) 1650118

B30 (2016) 1650200

B30 (2016) 1650093

B30 (2016) 1650086

B30 (2016) 1650017

B30 (2016) 1650143

B30 (2016) 1640017

B30 (2016) 1650017

B30 (2016) 1650052

B30 (2016) 1541008 B30 (2016) 1550247

B30 (2016) 1640022
Ma, D., see Yao, C.

Ma, K., see Zhang, Y.

B30 (2016) 1650015

Ma, L., see Liu, G.

Ma, P., see Gu, J.

Ma, Q., see Huang, L.

Ma, Q., see Wang, G.

Ma, W.-X., Zhou, Y. \& Dougherty, R., Lumptype solutions to nonlinear differential equations derived from generalized bilinear equations

Ma, W.-X., see Yang, J.-Y.

Ma, W.-X., see Zhang, L.

Ma, X., Zhang, Y., Ran, H. \& Zhang, Q., Segregation simulation of binary granular matter under horizontal pendulum vibrations

Ma, Z.-J., see Han, X.-Y.

Maaref, H., see Besahraoui, F.

Maaref, H., see Ilahi, B.

Mabuchi, H., see Hamerly, R.

Machado, P. C. M., Osório, F. A. P. \& Borges, A. $\mathrm{N}$., Inter-subband structure factor for a quasione-dimensional polaron gas

Mahdian, M., Arjmandi, M. B. \& Marahem, F., Chain mapping approach of Hamiltonian for FMO complex using associated, generalized and exceptional Jacobi polynomials

Mahesh, D. \& Mandal, S. $\mathrm{K}$., Multiferroicity in $\mathrm{ZnO}$ nanodumbbell/BiFe $\mathrm{O}_{3}$ nanoparticle heterostructures

Mahmood, A., see Basit, A.

Mahmood, A., see Ramay, S. M.

Mahmood, A., see Ullah, N.

Mahmood, Q., Faridi, A., Asif MahmoodRashid, M., Hassan, M. \& Noor, N. A., Electronic structure and magnetic properties of $\mathrm{Zn}_{1-x} T M_{x} \mathrm{Te} \quad(\mathrm{TM}=\mathrm{Fe}$, Co, Ni) for $0 \leq x \leq 1$ al-
B30 (2016) 1640031

B30 (2016) 1650143

B30 (2016) 1650165

B30 (2016) 1650167

B30 (2016) 1650023

B30 (2016) 1640018

B30 (2016) 1640028

B30 (2016) 1640029

B30 (2016) 1650214

B30 (2016) 1650172

B30 (2016) 1650145

B30 (2016) 1650210

B30 (2016) 1630014

B30 (2016) 1650218

B30 (2016) 1650107

B30 (2016) 1650074

B30 (2016) 1650221

B30 (2016) 1650020

B30 (2016) 1650137 
loys

Mahomed, F. M., see Carrim, A. H.

Mahomed, F. M., see Fatima, A.

Mahomed, K. S. \& Moitsheki, R. J., First integrals of generalized Ermakov systems via the Hamiltonian formulation

Mahudeswaran, A., Vivekanandan, J., Vijayanand, P. S., Kojima, T. \& Kato, S., A facile synthesis of poly(anilineco-o-bromoaniline) copolymer: Characterization and application as semiconducting material

Maiti, S. K., see Dey, M.

Malik, P., see Singh, A.

Malozovsky, Y. M., see Fan, J. D.

Mana, G., see Gervino, G.

Mandal, S. K., see Mahesh, D.

Marahem, F., see Mahdian, M.

Marselin, M. A. \& Jaya, N. V., Structural, optical and high pressure electrical resistivity studies of pure $\mathrm{NiO}$ and $\mathrm{Cu}$-doped $\mathrm{NiO}$ nanoparticles

Mason, D. P., see Fareo, A. G.

Mason, D. P., see Kokela, L. $\mathrm{N}$.

Maśka, M. M., see JanusZygmunt, I.

Md. Rahaman, Z., see Md. Rahman, A.

Md. Rahman, A., Md. Rahaman, Z. \& Md. Rahman, A., The structural, elastic, electronic and optical properties of $\mathrm{MgCu}$ under pressure: A firstprinciples study

Md. Rahman, A., see Md. Rahman, A.

ME. Barakat, M., Abou-Aly, A. I., Awad, R., Aly, N. S. \& Ibrahim, S., Excess conductivity analysis for
B30 (2016) 1650160

B30 (2016) 1640007

B30 (2016) 1640011

B30 (2016) 1640019

B30 (2016) 1650008

B30 (2016) 1650184

B30 (2016) 1650011

B30 (2016) 1642006

B30 (2016) 1541002

B30 (2016) 1650074

B30 (2016) 1650107

B30 (2016) 1650056

B30 (2016) 1640010

B30 (2016) 1640014

B30 (2016) 1642013

B30 (2016) 1650199

B30 (2016) 1650199

B30 (2016) 1650199
$\mathrm{Y}_{3-x} \mathrm{Nd}_{x} \mathrm{Ba}_{5-x} \mathrm{Ca}_{x} \mathrm{Cu}_{8} \mathrm{O}_{18-\delta}$ superconducting phase

B30 (2016) 1650115

Mehdiyeva, R., see Garibli, A.

B30 (2016) 1650040

Melnik, R., see Prabhakar, S.

B30 (2016) 1642003

Mendi, R. T., see Sarmazdeh, M. M.

B30 (2016) 1650117

B30 (2016) 1650236

B30 (2016) 1650086

Meng, Q., see Wang, $\mathrm{H}$

Meng, X. \& Huang, J., Fast phase transition of water molecules in a defective carbon nanotube under an electric field

B30 (2016) 1650019

Menteshashvili, M., see Grusha, I.

Meradji, H., see Benlamari, S.

B30 (2016) 1550260

B30 (2016) 1650003

Meslouhi, A., Amellal, H., Hassouni, Y., El Baz, M. \& El Allati, A., Quantum key distribution protocol using random bases

Meybodi, M. R., see Khomami, M. M. D.

B30 (2016) 1650061

B30 (2016) 1650042

Mi, X. W., see de Ouyang,

Mignani, R., see Cardone, F.

Min, Y., see Shen, J.-M.

Mishinova, G. N., see Vlaskina, S. I.

Mishra, A. C., Micromagnetic simulation of hysteresis loop of elliptic permalloy nanorings

Mizher, A. J., Raya, A. \& Villavicencio, C., Electric current generation in distorted graphene

Mo, J.-J., see Kong, X.-K.

Mogios, E., see Asimakopoulos, A.

Mohammad Alavi, S. \& Bagani, E., Electron mobility limited by scattering from threading dislocation lines within gallium nitride

Mohammadi Sabet, M. T., see Bordbar, G. H.

Mohammed, M. \& Sango, M., A tartar approach to periodic homogenization of linear hyperbolic
B30 (2016) 1650235

B30 (2016) 1550239

B30 (2016) 1550256

B30 (2016) 1642019

B30 (2016) 1650192

B30 (2016) 1550257

B30 (2016) 1650070

B30 (2016) 1541009

B30 (2016) 1650050

B30 (2016) 1650129 
stochastic partial differential equation

Mohsen-Nia, M., Abadian, F., Abadian, N., Dehkordi, K. M., Keivani, M. \& Abadyan, M., Analysis of cantilever NEMS in centrifugal-fluidic systems

Moitsheki, R. J., see Mahomed, K. S.

Molati, M. \& Khalique, C. M., Direct approach to a group classification problem: Fisher equation with time-dependent coefficients

Monti, M., see Cardone, F.

Moradi, A., Quantum nonlocal polarizability of spherical metal nanoparticles

Morinari, T., see Okabayashi, A.

Motamedinasab, A., see Afshar, D.

Mozume, T., see GonzálezFernández, J. V.

Muatjetjeja, B., Group classification and conservation laws of the generalized Klein-Gordon-Fock equation

Mubarak, A. A., see Hamioud, F.

Mubarak, A. A., The firstprinciple study of the electronic, optical and thermoelectric properties of $\mathrm{XTiO}_{3}(\mathrm{X}=\mathrm{Ca}$, Sr and Ba) compounds

Muhammad, R. \& Iqbal, Y., Structure-dielectric property relationship in $\mathrm{Nb}$ doped $\mathrm{Ca}_{4} \mathrm{La}_{2} \mathrm{Ti}_{5} \mathrm{O}_{17}$ ceramics

Muhammad, S., see Basit, A.

Mukherjee, P. K. \& Das, A. K., Fluctuation-induced dielectric permittivity in the isotropic phase of cholesteric liquid crystals

Murai, D., see Kaneko, S.

Muralidhar, M., see Koblis- chka, M. R.

B30 (2016) 1630017

B30 (2016) 1650221

B30 (2016) 1640020

Murtaza, G., see Basit, A.

Murtaza, G., see El Amine Monir, M.

B30 (2016) 1650034

B30 (2016) 1650137

Murtaza, G., see Ullah, N.

Murugan, R., see Rameshe, B.

B30 (2016) 1650148

Muzaffar, M. U. \& Khan, N. A., Effect of $\mathrm{CuO}_{2}$ planes on the structural and superconducting transport properties of [CuTl$12(n-1) n ; n=2,3,4]$ superconductor family

Muzaffar, M. U., see Khan, N. A.

B30 (2016) 1640021

B30 (2016) 1550239

Nakazawa, Y., see Imajo, S.

Nakazawa, Y., see Kruchinin, $\mathrm{S}$.

Nakazawa, Y., see Yamashita, $\mathrm{S}$.

B30 (2016) 1650048

B30 (2016) 1650226

B30 (2016) 1650026

B30 (2016) 1550248

B30 (2016) 1640023

B30 (2016) 1650031

B30 (2016) 1650141

B30 (2016) 1650104

B30 (2016) 1650221

B30 (2016) 1650053 B30 (2016) 1642010
Nalbandyan, M., see Lazaryan, $\mathrm{H}$.

Nam, N. D., see Hoi, B. D.

Nam, S. W., see Yoon, J. S.

Naqib, S. H., see Ali, M. A.

Nasir, M. T., see Ali, M. A.

Naumis, G. G., see GómezArias, W. A.

Nejatipour, H. \& Dadsetani, M., First principles study of the structural, electronic and optical properties of crystalline o-phenanthroline

Nekrasov, I. A., see Gavrichkov, V. A.

Neto, F. D., see Neto, M. A.

Neto, M. A., de Sousa, J. R., Padilha, I. T., Salmon, O. D. R., Viana, J. R. \& Neto, F. D., The magnetic susceptibility on the transverse antiferromagnetic Ising model: Analysis of the reentrant behavior

Nguyen, T. A., see Tran, H. P.

Nhan, N. T. T., Kien, P. H., Hung, P. K., Hong, N. V. \& San, L. T., About the diffusion mechanism in the silica liquid

Ni, Z., see Fang, Y.

Ning, F., see Zhong, S.
B30 (2016) 1650059

B30 (2016) 1550246

B30 (2016) 1650112

B30 (2016) 1650097 B30 (2016) 1642014

B30 (2016) 1642020

B30 (2016) 1642007

B30 (2016) 1650135

B30 (2016) 1650004

B30 (2016) 1650146

B30 (2016) 1650089

B30 (2016) 1650089

B30 (2016) 1550263

B30 (2016) 1650077

B30 (2016) 1650180

B30 (2016) 1630011

B30 (2016) 1630011

B30 (2016) 1650195

B30 (2016) 1650119

B30 (2016) 1650176 
Nishinari, K., see Tannai, T. Niu, S., see Jin, Y.

Nofeli, F., see Sarmazdeh, M. M.

Noor, N. A., see Mahmood, $\mathrm{Q}$.

Ocak, S. B., see Çağatay, B.

Okabayashi, A. \& Morinari, T., Anisotropic diffusion of conduction electron under antiferromagnetic spin configuration

Olayinka, A. S., see Adetunji, B. I.

Omanwar, S. K., see Taide, S. T.

Ong, L.-H., see Alrub, A. M.

Orekhovskaja, T. I., see Grushevskaya, H. V.

Orhan, Ö. \& Özer, T., Linearization properties, first integrals, nonlocal transformation for heat transfer equation

Örnek, O. \& Arinodotkan, N., Structural, electronic, elastic, thermodynamic and phonon properties of $\operatorname{LaX}(x=\mathrm{Cd}, \mathrm{Hg}$ and $\mathrm{Zn)}$ compounds in the B2 phase

Ortega-Gallegos, J., see González-Fernández, J. $\mathrm{V}$.

Osório, F. A. P., see Machado, P. C. M.

Ovchinnikov, S. G., see Gavrichkov, V. A.

Owaidat, M. Q., Asad, J. H. \& Tan, Z.-Z., On the perturbation of a uniform tiling with resistors

Ozebo, V. C., see Adetunji, B. I.

Özer, T., see Orhan, Ö.

Padhi, P. C., see Das, S.

Padilha, I. T., see Neto, M. A.

Pal, P. S., Saha, A. \& Jayannavar, A. M., Operational characteristics of singleparticle heat engines and refrigerators with time-
B30 (2016) 1650220

B30 (2016) 1650102

B30 (2016) 1650117

B30 (2016) 1650160

B30 (2016) 1650062

B30 (2016) 1650226

B30 (2016) 1650175

B30 (2016) 1650179

B30 (2016) 1650082

B30 (2016) 1642018

B30 (2016) 1640024

B30 (2016) 1650169

B30 (2016) 1550248

B30 (2016) 1650218

B30 (2016) 1650180

B30 (2016) 1650166

B30 (2016) 1650175

B30 (2016) 1640024

B30 (2016) 1650044

B30 (2016) 1630011 asymmetric protocol

Palanivel, B., see Rameshe, B.

Palmisano, C., see Gervino, $\mathrm{G}$.

Pang, W., see Huang, L.

Pang, W., see Wang, G.

Pantha, N., see Pokharel, S.

Papadimitriou, C., see Constantoudis, V.

Papageorgiou, H., see Constantoudis, V.

Park, D. J., Pradhan, P. \& Backman, V., Enhancing the sensitivity of mesoscopic light reflection statistics in weakly disordered media by interface reflections

Pavkov-Hrvojević, M. V., see Rutonjski, M. S.

Pchelkina, Z. V., see Gavrichkov, V. A.

Pedrosa, I. A., see de Melo, J. L.

Pei, H., see Li, H.

Peng, T., see Li, Q.

Peng, X., see Li, Q.

Peng, Y.-F., see Han, X.-Y.

Peng, Y.-X., see Wang, K.-J.

Petrovic, C., see Granstrom, C. R.

Petrucci, A., see Cardone, F.

Pirozhenko, I. G., see Bordag, M.

Pokharel, S., Pantha, N. \& Adhikari, N. P., Diffusion coefficients of nitric oxide in water: A molecular dynamics study

Polyakov, P. A., see Akimov, M. L.

Prabhakar, S. \& Melnik, R., Tuning g-factor of electrons through spin-orbit coupling in GaAs/AlGaAs conical quantum dots

Pradhan, P., see Park, D. J.

Prelovšek, P. \& Lenarčič, $\mathrm{Z}$., Charge relaxation and recombination in photoexcited Mott insulators

Pylyuk, I. V. \& Kozlovskii, M. P., Structural characteristics of a three-
B30 (2016) 1650219

B30 (2016) 1550246

B30 (2016) 1541002

B30 (2016) 1650167

B30 (2016) 1650023

B30 (2016) 1650205

B30 (2016) 1541005

B30 (2016) 1541005

B30 (2016) 1650155

B30 (2016) 1550251

B30 (2016) 1650180

B30 (2016) 1650022

B30 (2016) 1650072

B30 (2016) 1650212

B30 (2016) 1650212

B30 (2016) 1650172

B30 (2016) 1650088

B30 (2016) 1642002

B30 (2016) 1550239

B30 (2016) 1650120

B30 (2016) 1650205

B30 (2016) 1650081

B30 (2016) 1642003

B30 (2016) 1650155

B30 (2016) 1642015 
dimensional Ising-like system in the critical region

Qin, X.-K., Quantum switch in coupled-resonator array: Controlling singlephoton transport by the state of two-level system

Qin, X.-K., Role of superconductivity in superconducting transmission line resonator

Qin, Y., see Wang, J.

Qing, Z. Y., see Ding, D.

Qu, L., see Hou, Q.

$\mathrm{Qu}, \mathrm{P}$, Effect of crosssectional geometry on thermal conductivity of $\mathrm{Si}_{1-x} G e_{x}$ nanowires

Rai, D. P., see Chettri, S.

Ramay, S. M., Mahmood, A., Atiq, S. \& Al Hazaa, A. N., Study of divalent elements $(\mathrm{Mg}, \mathrm{Sr}$ and $\mathrm{Ba}$ )-doped $\mathrm{LaMnO}_{3}$ nano-manganites

Rameshe, B., Murugan, R. \& Palanivel, B., First principle study on electronic structure, structural phase stability, optical and vibrational properties of $\mathrm{Ba}_{2} \mathrm{ScMO}_{6}(\mathrm{M}=\mathrm{Nb}, \mathrm{Ta})$

Ramšak, A., see Kregar, A.

Ran, H., see Ma, X.

Ranjbar, M. \& Bahari, A., Phase-matching condition of four-wave mixing in cylindrical nonlinear optical media

Rao, F., see Zhong, S.

Rao, W.-F., see Wang, L.

Rashid, M., see Mahmood, Q.

Ray, A. K., see Satpathi, U.

Raya, A., see HernándezOrtiz, S.

Raya, A., see Mizher, A. J.

Raza, R., see Hussain, M. J.

Ren, H., see Gao, X.

Reng, X., see Liu, Y.

Reyes-Serrato, A., see Xiao, M.

Rezvanian, A., see Khomami, M. M. D.

\section{B30 (2016) 1650232}

B30 (2016) 1550262

B30 (2016) 1650163

B30 (2016) 1550253

B30 (2016) 1650028

B30 (2016) 1650215

B30 (2016) 1650108 B30 (2016) 1650078

B30 (2016) 1650020

B30 (2016) 1550246 B30 (2016) 1642016

B30 (2016) 1650214

B30 (2016) 1650182

B30 (2016) 1650176

B30 (2016) 1650116

B30 (2016) 1650160

B30 (2016) 1550266

B30 (2016) 1650084

B30 (2016) 1550257

B30 (2016) 1650161

B30 (2016) 1650157

B30 (2016) 1650085

B30 (2016) 1650106

B30 (2016) 1650042
Ridolfi, F., see Cardone, F.

Rodionov, V. E., see Vlaskina, S. I.

Roknuzzaman, M., see Ali, M. A.

Rosa, M., see Gandarias, M. L.

Rosada, A., see Cardone, F. Rosetto, F., see Cardone, F. Rouhi, S., see Ansari, R.

Rouhi, S., On the effect of vacancy defect on the mechanical properties of gallium nitride nanosheets

Rubin, P., see Kristoffel, N.

Rutonjski, M. S., PavkovHrvojević, M. V. \& Berović, M. B., Effects of frustration and cyclic exchange on the spin$1 / 2$ heisenberg antiferromagnet within the self-consistent spin-wave theory

Sabah, C., see Bakir, M.

Safari, N., see Baniasadi, F. Saha, A., see Pal, P. S.

Sahli, B., Bouafia, H., Abidri, B., Bouaza, A., Akriche, A., Hiadsi, S. \& Abdellaoui, A., Study of hydrostatic pressure effect on structural, mechanical, electronic and optical properties of $\mathrm{KMgF}_{3}, \quad \mathrm{~K}_{0.5} \mathrm{Na}_{0.5} \mathrm{MgF}_{3}$ and $\mathrm{NaMgF}_{3}$ cubic fluoroperovskites via ab initio calculations

Sahraei, N., see Baniasadi, F.

Saib, S., see Ferahtia, S.

Saidi, F., see Besahraoui, F.

Saka, E., see Sezer, N.

Sakiroglu, S., Linear and nonlinear optical absorption coefficients and refractive index changes in Morse quantum wells under electric field

Sala, V., see Cardone, F.

Salah, M., Abdel-Rahman, M., Badawi, E. A. \& Abdel-Rahman, M. A., Determination of the
B30 (2016) 1550239

B30 (2016) 1642019

B30 (2016) 1650089

B30 (2016) 1640012

B30 (2016) 1550239

B30 (2016) 1550239

B30 (2016) 1650007

B30 (2016) 1650151 B30 (2016) 1642017

B30 (2016) 1550251

B30 (2016) 1650133

B30 (2016) 1650174

B30 (2016) 1650219

B30 (2016) 1650230

B30 (2016) 1650174

B30 (2016) 1650147

B30 (2016) 1650145

B30 (2016) 1650030

B30 (2016) 1650209

B30 (2016) 1550239 
activation enthalpy for migration of dislocations in plastically deformed 8006 Al-alloy by positron annihilation lifetime technique

Salas, P., see Barragán, V. E.

Sali, I., Tabi, C. B., Ekobena, H. P. \& Kofané, T. C., Modulational instability in a biexciton molecular chain with saturable nonlinearity effects

Salmon, O. D. R., see Neto, M. A.

San, L. T., see Nhan, N. T. T.

Sánchez-Madrigal, S., see Hernández-Ortiz, S.

Sango, M. \& Tegegn, T. A., Harmonic analysis tools for stochastic magnetohydrodynamics equations in Besov spaces

Sango, M., see Ali, Z. I.

Sango, M., see Mohammed, M.

Santoro, E., see Cardone, F.

Saparbaev, T., see Yavidov, B.

Sari, H., see Al, E. B.

Sarmazdeh, M. M., Mendi, R. T., Zelati, A., Boochani, A. \& Nofeli, F., First-principles study of optical properties of InN nanosheet

Satpathi, U., Ghosh, S., Ray, A. K. \& Deo, P. S., Localization of electrons in internal frame

Serra, P., see Cuestas, E.

Sezer, N. \& Saka, E., Impact of hydrogen forming gas annealing on microwave properties of $\mathrm{Ba}\left(\mathrm{Zn}_{1 / 3} \mathrm{Ta}_{2 / 3}\right) \mathrm{O}_{3}$ dielectric ceramics

Sh. Fujii, see Kaneko, S.

Shahid, S. A., see Hussain, M. J.

Shahverdiev, E. M., Controlling chaos in some laser systems via variable coupling and feedback time delays
B30 (2016) 1550244

B30 (2016) 1630011

B30 (2016) 1650059

B30 (2016) 1650084

B30 (2016) 1640025

B30 (2016) 1640002

B30 (2016) 1640020

B30 (2016) 1550239

B30 (2016) 1650186

B30 (2016) 1650139

B30 (2016) 1650117

B30 (2016) 1550266

B30 (2016) 1650055

B30 (2016) 1650030

B30 (2016) 1642010

B30 (2016) 1650161

B30 (2016) 1650181
Shankar, A., see Chettri, S.

Shao, J.-X., see Wang, P.-P.

Shejwal, N. N., Anis, M., Hussaini, S. S. \& Shirsat, M. D., Investigation on structural, $U V$-visible, SHG efficiency, dielectric, mechanical and thermal behavior of 1-cystine doped zinc thiourea sulphate crystal for NLO device applications

B30 (2016) 1650078

B30 (2016) 1550250

B30 (2016) 1650159

Shen, J.-M., Liu, J., Min, Y. \& Zhou, L.-P., Some methods to regulate lowbias negative differential resistance in $\sigma$ barrier separating nanoscale molecular transport systems

B30 (2016) 1550256

Sherman, A., Strong coupling diagram technique for the three-band Hubbard model

Shi, D., see Wei, X.

Shi, H., Zhou, C., Zhang, C. \& Ye, E., Anions $(n, s)$ mono-doping and co-doping influences on electronic structures and optical properties of $\mathrm{InNbO}_{\mathbf{4}}$

Shi, W., see Kong, X.-K.

Shi, X. \& Xi, W., Synchronization and rhythm dynamics of a neuronal network consisting of mixed bursting neurons with hybrid synapses

B30 (2016) 1650091

Shi, X., Shu, H., Zhu, J., Wang, X., Dong, L., Zhao, L., Liang, S. \& Liu, R., Research on wave bandgaps in a circular plate of radial phononic crystal

B30 (2016) 1650060 B30 (2016) 1650070

B30 (2016) 1642004 B30 (2016) 1550258

Shi, X., see Li, Q.

Shi, Y., Chen, M., Huang, J., Gu, Y. \& Fan, D., Using priority growth orientation of crystallite of the Monte Carlo method to study the process of crystal nucleation and growth in liquid phase
B30 (2016) 1650162

B30 (2016) 1650212

B30 (2016) 1650014 
Shimakawa, K., see Freitas, R. J.

Shirsat, M. D., see Shejwal, N. N.

Shoaib, M., see Aziz, A.

Shu, H., see Shi, X.

Shu, W., see Li, H.

Shulitski, B. G., see Grushevskaya, H. V.

Si, K., see Wei, X.

Singh, A. K., see Gupta, V.

Singh, A., Malik, P. \& Jayoti, D., Observation of blue phase in chiral nematic liquid crystal and its stabilization by silica nanoparticles

Singh, N., see Das, N.

Singh, N., see Das, N.

Singh, R. J. \& Khan, S., Model of electron pairs in electron-doped cuprates

Siyouri, F., El Baz, M. \& Hassouni, Y., Role of Wigner function in studying quantum correlations

Sökmen, I., see Al, E. B.

Solís, M. A., see Barragán, V. E.

Song, A. L., see Wang, X. P.

Song, A., Wang, X., Chen, T., Jiang, P. \& Bao, K., Low-frequency bandgaps of two-dimensional phononic crystal plate composed of asymmetric double-sided cylinder stubs

Song, C., see Zhang, X.-K.

Song, H., Huang, S. \& Liu, $\mathrm{X}$., Stochastic resonance in two-frequency signal systems

Song, K. H., see de Ouyang, $\mathrm{S}$.

Song, X., Wang, Q. \& Fu, L., Pair-production in polychromatic light oscillating electric fields

Song, Y., see Hao, Y.

Song, Z.-J., see Liu, X.

Spagnolo, B., see Anashkina, E. I.

Spera, G., see Cardone, F.

Stone, M., Berry phase
B30 (2016) 1650075

B30 (2016) 1650159

B30 (2016) 1640005

B30 (2016) 1650162

B30 (2016) 1650072

B30 (2016) 1642018

B30 (2016) 1650142

B30 (2016) 1650198

B30 (2016) 1650011

B30 (2016) 1650071

B30 (2016) 1630015

B30 (2016) 1650170

B30 (2016) 1650187

B30 (2016) 1650139

B30 (2016) 1650099

B30 (2016) 1650203

B30 (2016) 1650029

B30 (2016) 1650093

B30 (2016) 1650113

B30 (2016) 1650235

B30 (2016) 1650035

B30 (2016) 1650216

B30 (2016) 1650130

B30 (2016) 1541003

B30 (2016) 1550239 and anomalous velocity of Weyl fermions and Maxwell photons

Sujanah, P., John Peter, A. \& Lee, C. W., Magnetic field induced trions in a Telluride-based II-VI material

Sulaiman, G. M., see Ismail, R. A.

Sun, Y.-P., see Xu, X.-X.

Svaiter, N. F., see Acosta Diaz, R.

Svechnikov, G. S., see Vlaskina, S. I.

Syed, W. A. A., see Hussain, M. J.

Tabi, C. B., see Sali, I.

Tahiri, N., see Jabar, A.

Taide, S. T., Ingle, N. B. \& Omanwar, S. K., Standard approach for energy transfer scheme and tunable emission for white light-emitting diodes ( $W$ LEDs)

Takesue, H., see Hamerly, $\mathrm{R}$.

Talha, N., Bouazza, B., Bouazza, A. G. \& Kadoun, A.-E.-D., Comparative calculations of electron transport properties in 6H-SiC using three and five valley models

Tan, H., see Gao, X.

Tan, Z.-Z., see Owaidat, M. Q.

Tang, B. \& Li, D.-J., Quantum breathers in $X X Z$ ferromagnetic chains with on-site easy-plane anisotropy

Tang, B., see Yang, R.

Tang, H., see Yao, C.

Tannai, T. \& Nishinari, K., Backward propagation of congestion in TASEP ladder-network

Tarasewicz, P., The generalized Lang-Firsov transformation and the threeand the four-fermion interactions in a lattice fermion model
B30 (2016) 1550249

B30 (2016) 1650069

B30 (2016) 1650094

B30 (2016) 1640027

B30 (2016) 1650207

B30 (2016) 1642019

B30 (2016) 1650161

B30 (2016) 1550244

B30 (2016) 1650233

B30 (2016) 1650179

B30 (2016) 1630014

B30 (2016) 1650153 B30 (2016) 1650157

B30 (2016) 1650166

B30 (2016) 1650100

B30 (2016) 1550243

B30 (2016) 1650015

B30 (2016) 1650220

B30 (2016) 1650124 
Tarasov, V. E., Poiseuille equation for steady flow of fractal fluid

Tegegn, T. A., see Sango, M.

Tehranchi, M. M., see Baniasadi, F.

Thapa, R. K., see Chettri, S.

Thomas, G. F., The topological molecule: Its finite fluxes, exchange stability and minimal surfaces

Thouless, D. J., see Kosterlitz, J. M.

Tian, C.-L., see Zhong, M.M.

Tian, J. \& Zhang, L., Contribution to modeling the viscosity Arrhenius-type equation for saturated pure fluids

Tian, J., see Zhang, C.

Tian, Z. \& Jia, L., A new bus lane on urban expressway with no-bay bus stop

Tinh, B. D. \& Hoc, N. Q., Transport coefficients and Nernst signal of typeII superconductors under magnetic field

Tong, A., Chen, L. \& Li, Y., Pulse duration dependence of atomic sequential double ionization by circular laser pulses

Torda, A., see Kong, S.-G.

Tran, H. P., Nguyen, T. A., Le, V. H. \& Dang, T. M. H., Phase structure of binary Bose-Einstein condensates at finite temperature

Tsantili, I. C., see Hristopulos, D. T.

Tulepbergenov, S., see Yavidov, B.

Ullah, N., Murtaza, G., Iqbal, M. A., Mahmood, A. \& Khenata, R., Computational study of $\quad \mathrm{Cu} 2 \mathrm{ZnSn}\left(\mathrm{X}_{1-x} \mathrm{Te}_{x}\right)_{4}$ $(X=\mathrm{S}$, Se) for optoelectronic applications

Ulloa-Castillo, N. A., see González-Fernández, J. V.
B30 (2016) 1650128

B30 (2016) 1640025

B30 (2016) 1650174

B30 (2016) 1650078

B30 (2016) 1650039

B30 (2016) 1630018

B30 (2016) 1650201

B30 (2016) 1650202

B30 (2016) 1650154

B30 (2016) 1550264

B30 (2016) 1550267

B30 (2016) 1650223

B30 (2016) 1550255

B30 (2016) 1650195

B30 (2016) 1541007

B30 (2016) 1650186

B30 (2016) 1650137

B30 (2016) 1550248
Ungan, F., see Al, E. B.

Urzagasti, D., Soliton interaction and further merging in normal-dispersion fiber lasers modeled with the cubic-quintic CGLE

Uslu, İ, see Kaya, A.

Usmanov, N. N., see Akimov, M. L.

Uzunov, D. I., Fluctuation effects in metastable states near first-order phase transitions

Vaizie, H., see Ahmad, S.

Valenti, D., see Anashkina, E. I.

Valenzuela, D., see Hernández-Ortiz, S.

Valizadeh, M. M., Anisotropic heisenberg form of RKKY interaction in the one-dimensional spin-polarized electron gas

van Dongen, P. G. J., see Freericks, J. K.

Van Hooydonk, G., Classical and Mexican hat-type potential energy curve for the hydrogen molecule from a confined Kratzer oscillator

Veríssimo, N. C., see Duarte, R. N.

Viana, J. R., see Neto, M. A. Vijayanand, P. S., see Mahudeswaran, A.

Villavicencio, C., see Mizher, A. J.

Vinish, Y. \& Fleurov, V., Finite width of the optical event horizon and enhancement of analog Hawking radiation

Vivekanandan, J., see Mahudeswaran, A.

Vlahovic, B., see Filikhin, I.

Vlaskina, S. I., Kruchinin, S. P., Ya. Kuznetsova, E., Rodionov, V. E., Mishinova, G. N. \& Svechnikov, G. S., Nanostructures in silicon carbide crystals and films

Wang, A., see Wang, G.
B30 (2016) 1650139

B30 (2016) 1650231

B30 (2016) 1650090

B30 (2016) 1650081

B30 (2016) 1650149

B30 (2016) 1650079

B30 (2016) 1541003

B30 (2016) 1650084

B30 (2016) 1650234

B30 (2016) 1642001

B30 (2016) 1650043

B30 (2016) 1550261

B30 (2016) 1630011

B30 (2016) 1650008

B30 (2016) 1550257

B30 (2016) 1650197

B30 (2016) 1650008

B30 (2016) 1642011

B30 (2016) 1642019

B30 (2016) 1650096 
Wang, B., see Li, H.

Wang, B., see Zhang, B.

Wang, C., see Liu, D.

Wang, F. H., see Lou, K.

Wang, G., Huang, L., Wang, Y., Pang, W. \& Ma, Q., Link community detection based on line graphs with a novel link similarity measure

Wang, G., Jin, W. \& Wang, A., The complete synchronization of coupled Morris-Lecar neurons with chemical synapses

Wang, G., see Huang, L.

Wang, G., see Yang, K.

Wang, G., see Yuan, G.

Wang, H. K., see Xu, C.

Wang, H., Xu, W., Lui, Z., Chao, Z. \& Meng, Q., Dependency of hydromechanical properties of monzonitic granite on confining pressure and fluid pressure under compression

Wang, H., see Dai, Y. Y.

Wang, H., see Gao, X.

Wang, H., see Kong, Y.

Wang, H., see Li, P.

Wang, H., see Li, Z.

Wang, H., see Xia, S.

Wang, J., Han, R., Wei, X., Qin, Y., Yu, H. \& Deng, B., Weak signal detection and propagation in diluted feed-forward neural network with recurrent excitation and inhibition

Wang, J., Xu, J. J. \& Zhang, L., Simulation on the aggregation process of spherical particle confined in a spherical shell

Wang, J., see Cao, D.-W.

Wang, J., see Liu, X.

Wang, J., see Li, D.

Wang, J., see Li, S.

Wang, J., see Wei, X.

Wang, J., see Wei, X.

Wang, K.-J., Peng, Y.X., Wang, L., Liu, L.H., Li, Z.-J., Xu, L., Wang, X.-J., Li, J.-B.
B30 (2016) 1650072

B30 (2016) 1650013

B30 (2016) 1650037

B30 (2016) 1650200

B30 (2016) 1650023

B30 (2016) 1650096

B30 (2016) 1650167

B30 (2016) 1650168

B30 (2016) 1650127

B30 (2016) 1650098

B30 (2016) 1650086

B30 (2016) 1550254

B30 (2016) 1650157

B30 (2016) 1650136

B30 (2016) 1650051

B30 (2016) 1650196

B30 (2016) 1650217

B30 (2016) 1550253

B30 (2016) 1650065

B30 (2016) 1650114

B30 (2016) 1650130

B30 (2016) 1650012

B30 (2016) 1550240

B30 (2016) 1650142

B30 (2016) 1550258
\& He, M.-D., Light transmission through a one-dimensional metallic grating covered by a reduced cytochrome $c$ molecule layer

Wang, L., Li, Q.-F., Yang, C.-H., Wei, Y.-L., Zhu, X.-F. \& Rao, W.-F., Density-functional investigation of the geometric and electronic structure of ethylene oxide adsorbed on $\mathrm{Si}(100)$

Wang, L., see Wang, K.-J.

Wang, L., see Yan, Y.

Wang, L.-L., see Xiao, W.$\mathrm{Z}$.

Wang, M., see Kong, Y.

Wang, M., see Xia, S.

Wang, P.-P., Shao, J.-X. \& Cao, Q.-L., Melting properties of Pt and its transport coefficients in liquid states under high pressures

Wang, Q., see Song, X.

Wang, T., Dai, W., Jiao, P. \& Wang, W., Identifying influential nodes in dynamic social networks based on degree-corrected stochastic block model

Wang, T.-Y., Cai, X.-Q. \& Zhang, R.-L., Faulttolerant symmetricallyprivate information retrieval

Wang, W., see Wang, T.

Wang, W.-J., see Liu, X.

Wang, X. P., Jiang, P. \& Song, A. L., Lowfrequency and tuning characteristic of band gap in a symmetrical doublesided locally resonant phononic crystal plate with slit structure

Wang, X. P., Jiang, P., Chen, T. N. \& Yu, K. P., Frequency characteristics of defect states in a twodimensional phononic crystal with slit structure

Wang, X., see Shi, X.
B30 (2016) 1650088

B30 (2016) 1650116

B30 (2016) 1650088

B30 (2016) 1650237

B30 (2016) 1650236

B30 (2016) 1650136

B30 (2016) 1650217

B30 (2016) 1550250

B30 (2016) 1650035

B30 (2016) 1650092

B30 (2016) 1650178

B30 (2016) 1650092

B30 (2016) 1650130

B30 (2016) 1650203

B30 (2016) 1650025 B30 (2016) 1650162 
Wang, X., see Song, A.

Wang, X.-J., see Wang, K.J.

Wang, Y., see Huang, L.

Wang, Y., see Wang, G.

Wang, Y., A unified model for two-lane lattice traffic flow

Wei, J. Y. T., see Granstrom, C. R.

Wei, J., Zhang, H., Wu, Z., He, J. \& Guo, Y., A novel crowd flow model based on linear fractional stable motion

Wei, X., Shi, D., Yu, H., Deng, B., Lu, M., Han, C. \& Wang, J., Input-output mapping reconstruction of spike trains at dorsal horn evoked by manual acupuncture

Wei, X., Si, K., Yi, G., Wang, J. \& Lu, M., Geometric propertiesdependent neural synchrony modulated by extracellular subthreshold electric field

Wei, X., see Wang, J.

Wei, Y.-L., see Wang, L.

Wei, Y.-M., see Liu, X.

Wen, W. Z., see Ding, D.

Weng, G.-Q., see Yang, X.$\mathrm{H}$.

Woelki, M., Queuing model of a traffic bottleneck with bimodal arrival rate

Wu, J. H., see Gao, N.

$\mathrm{Wu}, \mathrm{M}$., see $\mathrm{Wu}, \mathrm{S}$.

Wu, M., see Zhong, S.

Wu, S., Fan, K., Wu, M. \& Yin, G., Two-dimensional $\mathrm{MnO}_{2} /$ graphene hybrid nanostructures as anode for lithium ion batteries

Wu, Y., see Liu, H.

Wu, Z., see Wei, J.

Xenikos, D. G., see Asimakopoulos, A.

Xi, W., see Shi, X.

Xia, S., Liu, L., Kong, Y., Wang, H. \& Wang, M., A first-principles study of the effects of differ-
B30 (2016) 1650029

B30 (2016) 1650088

B30 (2016) 1650167

B30 (2016) 1650023

B30 (2016) 1650227

B30 (2016) 1642002

B30 (2016) 1650049

B30 (2016) 1550258

B30 (2016) 1650142

B30 (2016) 1550253

B30 (2016) 1650116

B30 (2016) 1650130

B30 (2016) 1650028

B30 (2016) 1650222

B30 (2016) 1541001

B30 (2016) 1650111

B30 (2016) 1650208

B30 (2016) 1650176

B30 (2016) 1650208

B30 (2016) 1650229

B30 (2016) 1650049

B30 (2016) 1541009

B30 (2016) 1650091 ent Al constituents on

$G a_{1-x} A l_{x} N$ nanowires

Xia, S., see Kong, Y.

Xia, W., see Yang, K.

Xiao, M. \& Reyes-Serrato, A., Analytic AharonovBohm rings: Currents readout from Zeeman spectrum

Xiao, S., Chen, X. \& Liu, Y., Totally asymmetric simple exclusion process with a single defect site on boundaries

Xiao, W.-Z., Meng, B., Xu, H.-Q., Chen, Q. \& Wang, L.-L., Nitrogen-induced magnetism in stannates from first-principles calculations

Xiao, Z., see Liu, Y.

Xie, L., see Chen, Y.

Xu, C., Li, Q., Liu, C. M., Duan, M. Y. \& Wang, H. K., Structural stabilities, elastic and electronic properties of chromium tetraboride from firstprinciples calculations

Xu, H.-Q., see Xiao, W.-Z.

$\mathrm{Xu}$, J. J., see Wang, J.

$\mathrm{Xu}$, L., see Wang, K.-J.

$\mathrm{Xu}, \mathrm{W}$., see Wang, $\mathrm{H}$.

Xu, X.-X. \& Sun, Y.-P., Mukherjee-ChoudhuryChowdhury spectral problem and the semi-discrete integrable system

$\mathrm{Xu}, \mathrm{Z}$., see Hou, Q.

$\mathrm{Xu}, \mathrm{Z}$., see Huang, Y.

Xue, K., see Yu, L.-W.

Ya. Kuznetsova, E., see Vlaskina, S. I.

Yamada, A., A study of the magnetic properties in the Hubbard model on the honeycomb lattice by variational cluster approximation

Yamamoto, Y., see Hamerly, R.

Yamashita, S., Yoshizumi, M., Akutsu, H. \& Nakazawa, Y., Rotational tunneling of methyl
B30 (2016) 1650217

B30 (2016) 1650136

B30 (2016) 1650168

B30 (2016) 1650106

B30 (2016) 1650083

B30 (2016) 1650236

B30 (2016) 1650085

B30 (2016) 1650006

B30 (2016) 1650098

B30 (2016) 1650236

B30 (2016) 1650065

B30 (2016) 1650088

B30 (2016) 1650086

B30 (2016) 1640027

B30 (2016) 1650001

B30 (2016) 1650118

B30 (2016) 1630013

B30 (2016) 1642019

B30 (2016) 1650158

B30 (2016) 1630014 
groups and the electronic heat capacity of $\mathrm{EtMe}_{3} \mathrm{Sb}\left[\mathrm{Pd}(\text { dmit })_{2}\right]_{2}$ under magnetic fields

Yamashita, S., see Imajo, S.

Yamashita, S., see Kruchinin, $\mathrm{S}$.

Yan, H., see Li, S.

Yan, J., see Chen, Z.

Yan, Y., Wang, L., Liu, M. \& Chen, X., Evolutions of fluctuation modes and inner structures of global stock markets

Yang, C., see Liu, Y.

Yang, C.-H., see Wang, L.

Yang, C.-J., Jin, L.-H. \& Gong, W.-J., Onedimensional quantum spin heterojunction as a thermal switch

Yang, H. J., see Yoon, J. S.

Yang, J.-Y. \& Ma, W.-X., Lump solutions to the BKP equation by symbolic computation

Yang, K., He, Y., Yao, L., Cheng, Y., Wang, G. \& Xia, W., First-principles study of the cubic $\mathrm{CaHfO}_{3}$ (001) surface

Yang, L., see Fang, Y.

Yang, L.-X., Jiang, J. \& Liu, X.-J., Influence of community structure on the synchronization of power network

Yang, M., see Liu, H.

Yang, P., Fan, D. P. \& Li, Y. X Improve beam quality of laser proton acceleration with funnel-shapedhole target

Yang, Q., see Liu, H.

Yang, R., Tang, B. \& Gao, T., A comparison of mechanical properties between $\mathrm{Al}$ and $\mathrm{Al}_{3} \mathrm{Mg}$

Yang, S., see Yuan, G.

Yang, T., see Dai, Y. Y.

Yang, X.-H., Zhang, H.-F., Ling, F., Cheng, Z., Weng, G.-Q. \& Huang, Y.-J., Link prediction based on local community proper- ties

Yao, C., Zhang, Y., Ma, D. \& Tang, H., Enhancing the coupling of a two-coil system using a superscatterer

B30 (2016) 1642020

B30 (2016) 1650057

B30 (2016) 1650010

B30 (2016) 1650237

B30 (2016) 1650085

B30 (2016) 1650116

B30 (2016) 1650027

B30 (2016) 1650146

B30 (2016) 1640028

B30 (2016) 1650168

B30 (2016) 1650119

B30 (2016) 1550252

B30 (2016) 1650033

B30 (2016) 1650045 B30 (2016) 1650033

B30 (2016) 1550243

B30 (2016) 1650127

B30 (2016) 1550254

Yao, L., see Yang, K.

Yao, N. \& Zheng, Z., Chimera states in spatiotemporal systems: Theory and Applications

Yar, A., see Basit, A.

Yarlagadda, S., see Lone, M. Q.

Yavidov, B., Djumanov, S. H., Saparbaev, T., Ganiyev, O., Zholdassova, S., Tulepbergenov, S. \& Kurbanov, U., The combined effect of lattice's uniaxial strains and electron-phonon interaction's screening on $T_{\mathrm{BEC}}$ of the intersite bipolarons

Ye, E., see Shi, H.

Yesilgul, U., see Al, E. B.

Yi, G., see Wei, X.

Yi, H., see Zhang, C.

Yin, C., see Zhou, Y.

Yin, G., see Wu, S.

Yin, J., see Zhang, S.

Ying, C., Bai, X., Du, Y., Zhao, E., Lin, L. \& Hou, Q., Structural optimization and physical properties of $\mathrm{TcB}_{3}$ and $\mathrm{MoB}_{3}$ at high-pressure: Firstprinciples

Yoon, J. S., Hong, S.-K., Nam, S. W. \& Yang, H. J., Phase correlation of ensemble of quantum emitters and timed Dicke state

Yoshizumi, M., see Yamashita, S.

Yousefi, R. \& Doorzad, L., nanotube FET with lightly doped drain and source

\section{B30 (2016) 1650222}

B30 (2016) 1650015

B30 (2016) 1650068

B30 (2016) 1650168 Hetero-junction carbon regions

B30 (2016) 1630002

B30 (2016) 1650221

B30 (2016) 1650063

B30 (2016) 1650186

B30 (2016) 1650060

B30 (2016) 1650139

B30 (2016) 1650142

B30 (2016) 1650154

B30 (2016) 1650095

B30 (2016) 1650208

B30 (2016) 1650046

B30 (2016) 1650131

B30 (2016) 1650146

B30 (2016) 1642007

Yu, C., see Liu, H.

$\mathrm{Yu}, \mathrm{H}$., see Li, S.

Yu, H., see Wang, J.

Yu, H., see Wei, X.
B30 (2016) 1650005

B30 (2016) 1650229

B30 (2016) 1550240

B30 (2016) 1550253

B30 (2016) 1550258 
Yu, J., see Cheng, Q.-B.

Yu, K. P., see Wang, X. P.

Yu, L., see Gao, N.

Yu, L.-W., Xue, K. \& Ge, M.-L., Yang-Baxter matrices associated with quantum information based on the topological basis

Yu, Y.-J., see He, J.-Q.

Yu, Z.-Y., see Kong, X.-K.

Yu. Ovchinnikov, N., Superconductors with spinorbit interactions

Yuan, G., Bao, X., Yang, S., Wang, G. \& Chen, S., Dynamics of pulses and spiral waves in excitable media with an anomalous diffusion

Yuan, J., see Zhou, N.

Zaaboub, Z., see Ilahi, B.

Zahedi, E., Babaie, M. \& Bahmanpour, H., Adsorption properties of boroxol ring doped zigzag boron nitride nanotube toward NO molecule using DFT

Zaheri, A. H. M., Eigen function and corresponding eigen values of charge carriers in $V$-grooves quantum wires with variable width

Zelati, A., see Sarmazdeh, M. M.

Zeng, X., see Zhou, N.

Zeng, Y.-Z. \& Zhang, N., New full velocity difference model considering the driver's heterogeneity of the disturbance risk preference for carfollowing theory

Zeng, Z., see Dress, A.

Zhai, Y., see Gu, J.

Zhang, B., Wang, B. \& Liu, Q., Melting curves of $\mathrm{Cu}$, $\mathrm{Pt}, \mathrm{Pd}$ and $\mathrm{Au}$ under high pressures

Zhang, C., Yi, H. \& Tian, J., Lielmezs-Herrick correlation for the temperaturedependent surface tension of hydrocarbons
B30 (2016) 1650213

B30 (2016) 1650025

B30 (2016) 1650111

B30 (2016) 1630013

B30 (2016) 1550265

B30 (2016) 1650070

B30 (2016) 1650183

B30 (2016) 1650127

B30 (2016) 1650152

B30 (2016) 1650210

B30 (2016) 1650101

B30 (2016) 1650103

B30 (2016) 1650117

B30 (2016) 1650152

B30 (2016) 1550241

B30 (2016) 1630001

B30 (2016) 1650165

B30 (2016) 1650013

B30 (2016) 1650154
Zhang, C., see Shi, H.

Zhang, C., see Zhou, N.

Zhang, F.-D., see He, J.-Q.

Zhang, H. W., see Zhang, S.

Zhang, H., see Wei, J.

Zhang, H.-F., see Yang, X.H.

Zhang, L. L., see Li, Z. J.

Zhang, L., Khalique, C. M. \& Ma, W.-X., Classifying bilinear differential equations by linear superposition principle

Zhang, L., see Tian, J.

Zhang, L., see Wang, J.

Zhang, M., see Feng, Y. L.

Zhang, M.-H., see Zhang, Y.-H.

Zhang, N., see Zeng, Y.-Z.

Zhang, Q., see Ma, X.

Zhang, Q., see Zhang, X.-K.

Zhang, R.-L., see Wang, T.Y.

Zhang, S., Yin, J., Zhang, H. W. \& Chen, B. S., Multiobjective optimization of two-dimensional phoxonic crystals with multilevel substructure scheme

Zhang, W., see Lim, C.

Zhang, X.-K., Song, C., Jia, J., Lu, Z.-L. \& Zhang, Q., An improved label propagation algorithm based on the similarity matrix using random walk

Zhang, Y. \& Ma, K., The triple Wronskian solutions to the variable-coefficient Manakov model

Zhang, Y., see Cao, D.-W.

Zhang, Y., see Ma, X.

Zhang, Y., see Yao, C.

Zhang, Y., see Zhu, Y.

Zhang, Y.-H. \& Zhang, M.H., Hall and Nernst effects in monolayer $\mathrm{MoS}_{2}$

Zhang, Z. D., see Dai, Y. Y.

Zhang, Z., see Liu, H.

Zhao, C. J., see Cheng, W. D.

Zhao, C., see Hou, Q.

Zhao, C., see Hou, Q.

Zhao, E., see Ying, C.

Zhao, H., see Dai, Y.
B30 (2016) 1650060

B30 (2016) 1650152

B30 (2016) 1550265

B30 (2016) 1650046

B30 (2016) 1650049

B30 (2016) 1650222

B30 (2016) 1550242

B30 (2016) 1640029

B30 (2016) 1650202

B30 (2016) 1650065

B30 (2016) 1650067

B30 (2016) 1650041

B30 (2016) 1550241

B30 (2016) 1650214

B30 (2016) 1650093

B30 (2016) 1650178

B30 (2016) 1650046 B30 (2016) 1541006

B30 (2016) 1650093

B30 (2016) 1640031

B30 (2016) 1650114

B30 (2016) 1650214

B30 (2016) 1650015

B30 (2016) 1650193

B30 (2016) 1650041

B30 (2016) 1550254

B30 (2016) 1650229

B30 (2016) 1650052

B30 (2016) 1650215

B30 (2016) 1650001

B30 (2016) 1650131

B30 (2016) 1640009 
Zhao, H., see Feng, S.

Zhao, L., see Shi, X.

Zhao, M., see Chen, X. X.

Zhao, P., see Luo, L.

Zhao, X., see Lou, K.

Zhao, X.-M., see Cheng, Q.B.

Zheng, D.-Q., see Li, J.

Zheng, L., see Ding, D.

Zheng, Z., see Yao, N.

Zholdassova, S., see Yavidov, B.

Zhong, M.-M., Huang, C. \& Tian, C.-L., The structural stabilities, mechanical properties and hardness of chromium tetraboride: Compared with low- $b$ borides

Zhong, S., Ning, F., Rao, F., Lei, X., Wu, M. \& Zhou, L., First-principles study of nitrogen and carbon monoxide adsorptions on silicene

Zhou, C., see Shi, H.

Zhou, H.-F., see Dong, H.$\mathrm{N}$.

Zhou, J., see Li, H.

Zhou, L., see Zhong, S.

Zhou, L., see Zhou, N.

Zhou, L.-P., see Shen, J.-M.
B30 (2016) 1650176

B30 (2016) 1650194 B30 (2016) 1650060

B30 (2016) 1642005

B30 (2016) 1650162

B30 (2016) 1650068

B30 (2016) 1640017

B30 (2016) 1650200

B30 (2016) 1650213

B30 (2016) 1650194

B30 (2016) 1650028

B30 (2016) 1630002

B30 (2016) 1650186

B30 (2016) 1650201

B30 (2016) 1650204

B30 (2016) 1650072

B30 (2016) 1650176

B30 (2016) 1650152

B30 (2016) 1550256
Zhou, N., Zhang, C., Zeng, X., Yuan, J. \& Zhou, L., A molecular dynamics study of the growth rate of $\mathrm{SiC}$ crystal and its dependence on the temperature

B30 (2016) 1650152

Zhou, Y. B., see de Ouyang, $\mathrm{S}$.

Zhou, Y. \& Yin, C., Timedependent Kramers escape rate in overdamped system with power-law distribution

Zhou, Y., see Guo, L.

Zhou, Y., see Ma, W.-X.

Zhu, J., see Feng, Y. L.

Zhu, J., see Shi, X.

Zhu, W., see Li, J.

Zhu, X.-F., see Wang, L.

Zhu, Y., Zhang, Y., Li, Y. \& Hu, Z., Spiral spectrum of multi-modified BesselGaussian schell beams in non-Kolmogorov turbulence

Zia, M. F., see Ilahi, B.

Zipper, E., see JanusZygmunt, I.

Zolotovsky, A., see Kruchinin, $\mathrm{S}$.

Zuevsky, A., Cluster algebras based on vertex operator algebras
B30 (2016) 1650095

B30 (2016) 1650193

B30 (2016) 1650235

B30 (2016) 1650173

B30 (2016) 1640018

B30 (2016) 1650067

B30 (2016) 1650162

B30 (2016) 1640015

B30 (2016) 1650116

B30 (2016) 1650210

B30 (2016) 1642013

B30 (2016) 1642020

B30 (2016) 1640030 